\title{
$\beta$-Klotho deficiency protects against obesity through a crosstalk between liver, microbiota, and brown adipose tissue
}

\author{
Emmanuel Somm, ${ }^{1}$ Hugues Henry, ${ }^{2}$ Stephen J. Bruce, ${ }^{2}$ Sébastien Aeby, ${ }^{3}$ Marta Rosikiewicz, ${ }^{3}$ \\ Gerasimos P. Sykiotis, ${ }^{1}$ Mohammed Asrih, ${ }^{1}$ François R. Jornayvaz, ${ }^{1}$ Pierre Damien Denechaud, ${ }^{4}$ \\ Urs Albrecht, ${ }^{5}$ Moosa Mohammadi, ${ }^{6}$ Andrew Dwyer, ${ }^{1}$ James S. Acierno Jr., ${ }^{1}$ Kristina Schoonjans, \\ Lluis Fajas, ${ }^{4}$ Gilbert Greub, ${ }^{3}$ and Nelly Pitteloud ${ }^{1}$ \\ 'Service of Endocrinology, Diabetology and Metabolism, Lausanne University Hospital; Department of Physiology, \\ Faculty of Biology and Medicine, University of Lausanne, Lausanne, Switzerland. ${ }^{2}$ Clinical Chemistry Laboratory, \\ Lausanne University Hospital, Lausanne, Switzerland. Institute of Microbiology, Lausanne University Hospital, \\ Lausanne, Switzerland. ${ }^{4}$ Department of Physiology, Faculty of Biology and Medicine, University of Lausanne, Lausanne, \\ Switzerland. ${ }^{5}$ Department of Biology, Unit of Biochemistry, University of Fribourg, Fribourg, Switzerland. ${ }^{6}$ Department of \\ Biochemistry and Molecular Pharmacology, New York University School of Medicine, New York, New York, USA. 'Institute \\ of Bioengineering, Ecole Polytechnique Fédérale de Lausanne, Lausanne, Switzerland.
}

$\beta$-Klotho (encoded by $K / b$ ) is the obligate coreceptor mediating FGF21 and FGF15/19 signaling. $K^{\prime} b^{-1-}$ mice are refractory to beneficial action of pharmacological FGF21 treatment including stimulation of glucose utilization and thermogenesis. Here, we investigated the energy homeostasis in $\mathrm{KI}^{-/-}$mice on high-fat diet in order to better understand the consequences of abrogating both endogenous FGF15/19 and FGF21 signaling during caloric overload. Surprisingly, $\mathrm{Klb}^{-/-}$mice are resistant to diet-induced obesity (DIO) owing to enhanced energy expenditure and BAT activity. $\mathrm{KIb}^{-/-}$mice exhibited not only an increase but also a shift in bile acid (BA) composition featured by activation of the classical (neutral) BA synthesis pathway at the expense of the alternative (acidic) pathway. High hepatic production of cholic acid (CA) results in a large excess of microbiotaderived deoxycholic acid (DCA). DCA is specifically responsible for activating the TCR5 receptor that stimulates BAT thermogenic activity. In fact, combined gene deletion of Klb and Tgr5 or antibiotic treatment abrogating bacterial conversion of CA into DCA both abolish DIO resistance in $\mathrm{Kl}^{-/-}$mice. These results suggested that DIO resistance in $\mathrm{KI}^{-/-}$mice is caused by high levels of DCA, signaling through the TCR5 receptor. These data also demonstrated that gut microbiota can regulate host thermogenesis via conversion of primary into secondary BA. Pharmacologic or nutritional approaches to selectively modulate BA composition may be a promising target for treating metabolic disorders.

Conflict of interest: The authors have declared that no conflict of interest exists.

Submitted: November 28, 2016 Accepted: March 7, 2017 Published: April 20, 2017

Reference information: JCI Insight. 2017;2(8):e91809. https:// doi.org/10.1172/jii.insight.91809.

\section{Introduction}

$\beta$-Klotho $(K l b)$ has been identified due to its homology with the $\alpha$-klotho $(K \downarrow)$ gene (1). In contrast to $\alpha$-klotho, $\beta$-klotho is only found as a membrane protein and is abundantly expressed in peripheral metabolic tissues (1).

$\beta$-Klotho forms a binary complex with FGF receptor 1c (FGFR1c) and functions as an obligate coreceptor for FGF21 in vitro (2, 3). FGF21 is induced in the liver following nutritional stress (4). FGF21 stimulates glucose uptake and lipolysis in adipose tissue $(5,6)$ and activates thermogenesis $(7,8)$, thus making it of significant interest as a biologic drug to treat metabolic diseases. A Klb-deficient mouse model has been used to assess potential residual FGF21 activity in vivo. $\mathrm{Klb}^{-/-}$mice are fully resistant to FGF21-induced intracellular signaling and thus do not exhibit a physiologic response to exogenous FGF21 administration (9), confirming the vital role of $\beta$-klotho in the FGF21 signaling machinery in vivo. Similarly, whole-body $\mathrm{Klb}^{-/-}$mice overexpressing $\mathrm{Fgf21}$ in the liver fail to elicit FGF21 action (10). Several studies using tissuespecific $\mathrm{Klb}^{-1-}$ mice have revealed the target tissues of FGF21 action (10-13). Notably, mice with an adipose tissue-specific deficiency in $\beta$-klotho lose the acute insulin-sensitizing effect of FGF21 (10), whereas mice with a brain-specific deficiency in $\beta$-klotho lose the thermogenic effect of FGF21 (13). 
$\beta$-Klotho also forms a binary complex with FGFR4 and functions as an obligate coreceptor for FGF15 (FGF19 ortholog in humans) (14, 15). FGF15 is an ileum-derived hormone induced by eating and activation of the nuclear bile acid (BA) receptor FXR (16). Once released in the portal circulation, FGF15 binds to the FGFR4/ $\beta$-klotho complex in the liver, and triggers a reduction in the expression of Cyp7a1, the first and rate-limiting enzyme of the BA synthesis pathway (16).

Under basal conditions, $\mathrm{Klb}^{-/-}$mice exhibit a small gallbladder, with an increased BA pool and elevated fecal BA excretion (17). $\mathrm{Klb}^{-/-}$mice are protected against gallstone formation but exhibit few changes in the enterohepatic circulation of BA (17). Recent data demonstrate that reexpression of $K l b$ specifically in hepatocyte reverses the lipid dysregulations and normalizes BA metabolism in $\mathrm{Klb}^{-/-}$mice (18). $\mathrm{Klb}^{-/-}$mice exhibited a normal energy balance on standard chow diet $(17,18)$.

Here, we characterize the energy homeostasis resulting from a global $\beta$-klotho deficiency, under hypercaloric feeding conditions (i.e., high-fat diet [HFD]). Surprisingly, $\mathrm{Klb}^{-/-}$mice are resistant to diet-induced obesity (DIO) despite the absence of endogenous FGF21 and FGF15 signaling. A key role in this process is due to changes in BA composition, characterized by increased levels of the secondary BA deoxycholic acid (DCA), which is produced by microbiota from hepatic cholic acid (CA) and signals through the membrane BA receptor TGR5 in the brown adipose tissue (BAT) to enhance thermogenesis.

\section{Results}

$K_{l} b^{-1-}$ mice are resistant to DIO due to increased thermogenesis. To explore whether Klb deficiency impacts the response to caloric overload, we studied the metabolic parameters of $\mathrm{Klb}^{-1-}$ mice following 8 weeks of HFD. Before HFD initiation, body weights (BWs) of $\mathrm{Klb}^{-/-}$mice on a pure C57BL/6J background were approximately $15 \%$ lower than WT littermates at 10 weeks of age (Figure 1A, first time point). This reduced $\mathrm{BW}$ in basal conditions was similarly reported on a mixed genetic background, reflecting an isometric growth limitation with no change in adiposity $(17,18)$. $K l b^{-1-}$ mice gain less weight than WT on HFD (Figure 1A), exhibiting a massive limitation in fat mass accretion (Figure 1B). Consequently, fat proportion was massively reduced in the HFD-fed $\mathrm{Klb}^{-/-}$mice compared with HFD-fed WT mice (Figure 1C), resulting in significantly lower circulating leptin levels (Figure 1D). A lower epididymal white adipose tissue (eWAT)/BW ratio (Figure $1 \mathrm{E}$ ) and a reduction in adipocyte hypertrophy (Figure $1 \mathrm{~F}$ ) were also observed in HFD-fed $\mathrm{Klb}^{-/-}$mice. Gene expression studies in epididymal, perirenal, and inguinal WAT revealed similar patterns of regulation within the different WAT depots (Supplemental Figure 1; supplemental material available online with this article; https://doi.org/10.1172/jci.insight.91809DS1). This included increased expression levels of lipase ( $\mathrm{Atgl}, \mathrm{Hsl}$, and $\mathrm{Lpl}$ ) and adiponectin (Adipoq) as well as decreased levels of proinflammatory cytokine transcripts (in particular Tnf). In contrast, Ucp $1 \mathrm{mRNA}$ levels were decreased in eWAT and showed a trend towards a decrease in the other WAT depots, excluding a browning process. Circulating insulin levels were significantly lower (Figure 1G) and glucose tolerance was ameliorated (Figure $1 \mathrm{H}$ ) in $\mathrm{HFD}$-fed $\mathrm{Klb}^{-/-}$mice, probably reflecting their decreased adiposity. HFD-fed $\mathrm{Klb}^{-/-}$mice have a similar daily food intake compared with WT, and even a slightly increased food ingestion when reported relative to their metabolic BW (Figure 1I). No change was observed in hypothalamic gene expression of orexigenic/anorexigenic neuropeptides or inflammatory markers (Figure $1 \mathrm{~J}$ ). Evaluation of the energy content remaining in the feces showed the trend to be lower in HFD-fed $\mathrm{Klb}^{-/-}$mice compared with HFD-fed WT mice (Figure $1 \mathrm{~K}$ ). Taken together, these results excluded the possibility that a modification in food intake or nutrient assimilation underlies the resistance to DIO. In contrast, we observed significantly higher consumption of $\mathrm{O}_{2}\left(\mathrm{VO}_{2}\right)$ and production of $\mathrm{CO}_{2}\left(\mathrm{VCO}_{2}\right)$ (during both light and dark phases) in HFD-fed $\mathrm{Klb}^{-/-}$mice (Figure 2, A, B, D, and E). This increase in energy expenditure occurred in the setting of unchanged respiratory exchange ratio (RER) (Figure 2C) and spontaneous physical activity (Figure 2F). Therefore, we characterized the BAT of HFD-fed $\mathrm{Klb}^{-1-}$ mice. With a slight reduction in its relative mass (Figure $2 \mathrm{G}$ ), this tissue shows multilocular lipid droplets and an intense cytoplasmic staining compared with prominent unilocular lipid accumulation in BAT of HFD-fed WT mice (Figure 2H). Further, we observed increased expression levels of thermogenic and fat-oxidation-related genes (Ucp1, Ucp3, Dio2, Elovl3, Pgc1a, and Cidea) (Figure 2I), consistent with increased thermogenic activity of BAT in HFD-fed $\mathrm{Klb}^{-/-}$mice.

$\mathrm{Klb}^{-/-}$mice show a modified BA composition. Given the key role of FGF21/FGFR1c/ $\beta$-klotho signaling in fat oxidation, glucose uptake, and energy homeostasis, the resistance of $\mathrm{Klb}^{-/-}$mice to DIO was an unexpected observation. Further, these findings raised the question of the underlying mechanism(s). Since 
A

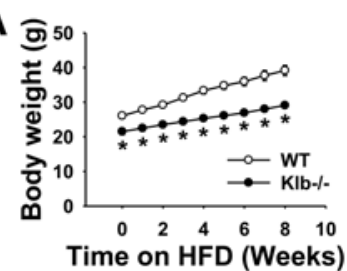

B
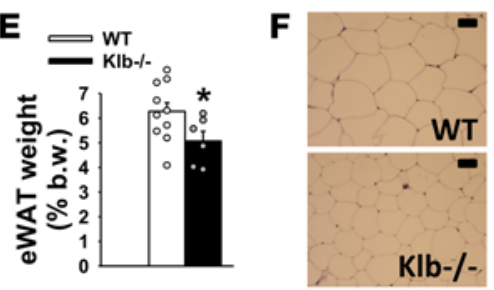

G

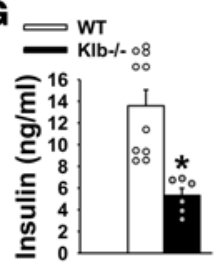

I

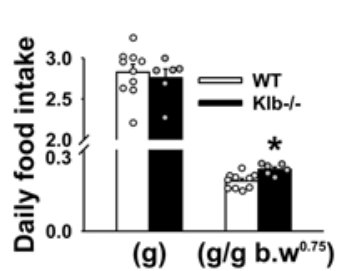

H

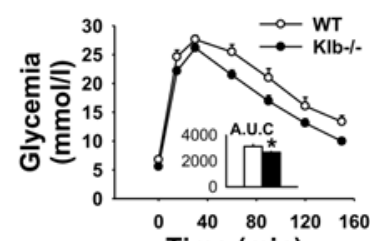

c $={ }_{\text {KIb-l-1 }}^{\text {wT }}$

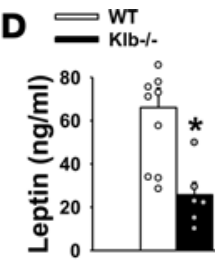

Time (min)

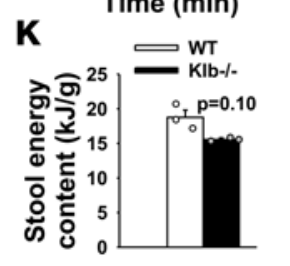

Figure 1. Resistance to high-fat diet-induced obesity in $\mathrm{KI}^{-/-}$mice. (A) Body weight curve. HFD, high-fat diet. (B) Lean and fat mass. (C) Fat proportion. (D) Circulating leptin levels. (E) Epididymal white adipose tissue (eWAT) weight. (F) H\&E staining of eWAT sections. Scale bars: $100 \mu \mathrm{m}$. (G) Circulating insulin levels. (H) Glucose tolerance test. (I) Daily food intake. (J) Hypothalamic mRNA levels of orexigenic/anorexigenic neuropeptides and inflammatory markers. (K) Stool energy content. Results are expressed as the mean \pm SEM. $n=5-6 \mathrm{KI}^{-1-}$ and $n=9-10$ WT male mice on HFD per group. For K, each individual value represents a pool of 2 to 4 mice. ${ }^{*} P<0.05$ versus WT determined by unpaired $t$ test. Individual values not represented (outside the range of the $y$ axis) in $\mathbf{D}$ (122 for leptin in WT group).

$\beta$-klotho is known to participate in a negative feedback loop that represses hepatic BA synthesis $(16,17)$, we investigated whether $K l b$ deficiency could also qualitatively impact BA homeostasis. Cyp7a1, the rate-limiting enzyme in BA synthesis, was highly overexpressed in the liver of $\mathrm{Klb}^{-1-}$ mice (Figure $3 \mathrm{~A})$, as previously reported (17). Cyp 861 (encoding sterol 12- $\alpha$-hydroxylase) was also upregulated, while Cyp7b1 and Cyp27al (driving the alternative [acidic] BA synthesis) were underexpressed compared with WT mice (Figure 3B). This specific transcriptional pattern of BA enzymes led us to investigate differences in hepatic primary BA production and the circulating BA composition. Primary BAs are directly synthesized by the liver and converted into secondary BAs by bacterial action in the colon. In the liver, tauro- $\beta$-muricholic acid (T- $\beta \mathrm{MCA}$ ) levels were decreased in $\mathrm{Klb}^{-1-}$ mice (Figure 3C), confirming the downregulation of the alternative pathway. Plasma levels of both primary taurocholic acid (T-CA) and its secondary derivative deoxycholic acid (conjugated [T-DCA] and unconjugated [DCA] forms) were dramatically increased in $\mathrm{Klb}^{-/}$mice (Figure 3, D and E). The global composition of circulating BAs was substantially modified between genotypes, with a predominance of T-CA and T-DCA in $\mathrm{Klb}^{-1-}$ mice (Figure 3F).

$\mathrm{Klb}^{-1-}$ mice are protected against hepatosteatosis. The massive change in BA levels and composition in $\mathrm{Klb}^{-1-}$ mice led us to investigate further hepatic parameters. While liver weight was not changed in HFD-fed $\mathrm{Klb}^{-1}$ mice (Figure 4A), hepatic histological sections (Figure 4B) and triglyceride content (Figure 4C) revealed lower hepatic lipid storage in $\mathrm{Klb}^{-/-}$versus WT mice on HFD. Further, plasma levels of total, HDL-, and LDL-cholesterol were also significantly lower in HFD-fed $\mathrm{Klb}^{-/-}$mice (Figure 4D). Overexpression of the ligand $\mathrm{Fg} 21$ and reduced expression of the receptor Fgfr4 were observed in the absence of $K l b$ (Figure 4E). Hepatic gene expression of key transcriptional regulators involved in lipid and BA metabolism (Srebp1c, Shp, and Lrh1) was strongly reduced in HFD-fed $\mathrm{Klb}^{-/-}$mice (Figure 4F). A concomitant downregulation of enzymes responsible for fatty acid synthesis (Acaca, Fasn, and $S c d 1$ ) and fatty acid oxidation (Cpt1a) was also observed in the liver of HFD-fed $\mathrm{Klb}^{-/-}$mice (Figure 4G). In line with their reduced hepatic triglyceride storage, HFD-fed $\mathrm{Klb}^{-/-}$mice showed higher Atgl and lower Dgat2 expression levels (Figure 4G), indicating stimulation of triglyceride breakdown and inhibition of triglyceride synthesis. Further, hepatic gene expression levels of enzymes responsible for cholesterol synthesis (Hmgcr and Hmgcs1) were higher in the HFD-fed $\mathrm{Klb}^{-1-}$ mice (Figure 4H), highlighting the activation of cholesterogenesis in the context of derepressed BA synthesis. Surprisingly, despite their lower hepatic lipid storage, the HFD-fed $\mathrm{Klb}^{-/-}$mice exhibited higher expression of proinflammatory cytokines ( $\mathrm{Tnf}$, $I l 1 b$, and Ilb) and lower expression of antiinflammatory marker (Nfkbia) in the liver (Figure 4I).

Resistance of $\mathrm{Klb}^{-1-}$ mice to DIO is Tgr5 dependent. Previous reports demonstrated that nutritional supplementation with BA during the consumption of HFD prevents the onset of obesity through activation of the membrane BA receptor (TGR5) in the BAT (19). Thus, to assess whether the change in plasma BA composition could be the mechanism involved in stimulating BAT and energy expenditure in the absence of $\beta$-klotho, we generated and phenotyped a double-KO mouse model ( $K l b^{-1-} \mathrm{Tgr} 5^{-1-}$ mice). At 10 weeks of age, $\mathrm{Klb}^{-1-} \mathrm{Tgr5^{-/- }}$ mice were similar to $\mathrm{Klb}^{-1-}$ mice, exhibiting a significantly lower BW compared with WT (Figure $5 \mathrm{~A}$, first time point). After 5 weeks on $\mathrm{HFD}$, the body weight evolution in $\mathrm{Klb}^{-1-} \mathrm{Tgr5^{-/ }}$ mice was 
A

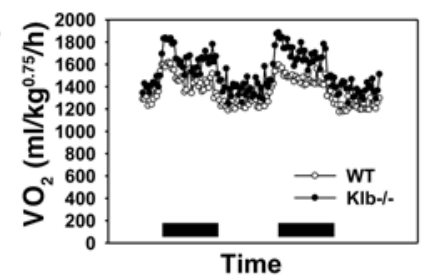

D

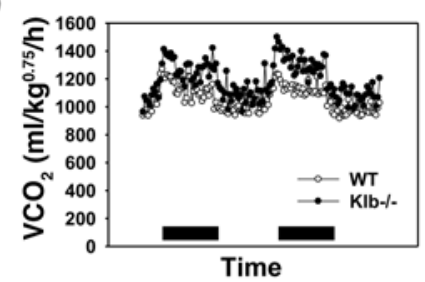

B

\section{,}

$\mathbf{E}$

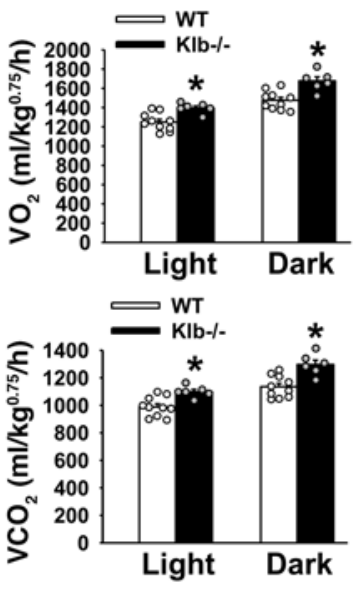

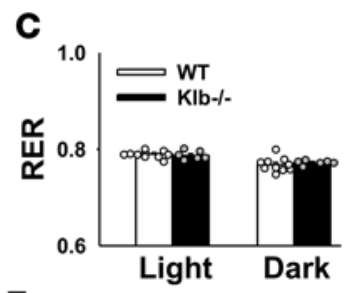

$\mathbf{F}$

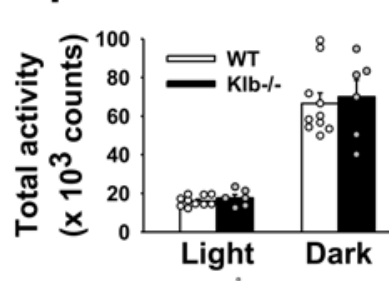

G

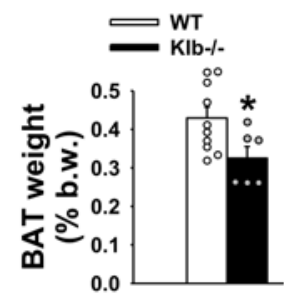

H

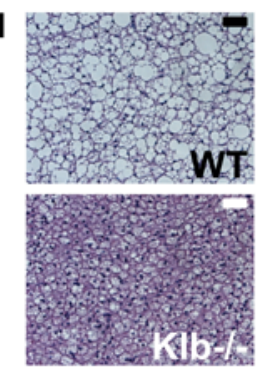

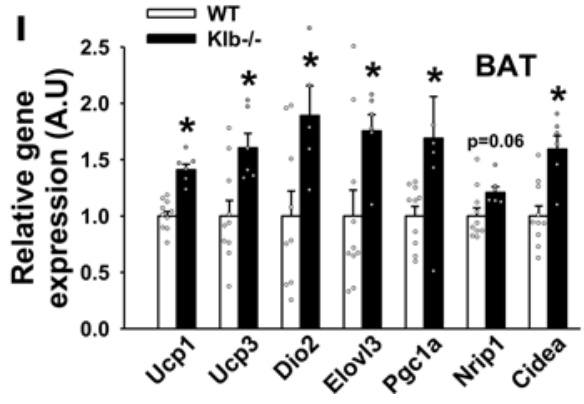

Figure 2. Increased energy expenditure in $\mathrm{KI}^{-/-}$mice on high-fat diet (HFD). (A) $\mathrm{O}_{2}$ consumption $\left(\mathrm{VO}_{2}\right)$ curves representing 2 consecutive days. (B) Mean $\mathrm{VO}_{2}$ during light and dark phases. (C) Respiratory exchange ratio $\left(\mathrm{RER}=\mathrm{VCO}_{2} / \mathrm{VO}_{2}\right.$, where $\mathrm{VCO}_{2}$ is $\mathrm{CO}_{2}$ production) during light and dark phases. (D) $\mathrm{VCO}_{2}$ curves representing 2 consecutive days. (E) Mean $\mathrm{VCO}_{2}$ during light and dark phases. (F) Spontaneous total activity (counts) during light and dark phases. (G) Brown adipose tissue (BAT) weight. (H) H\&E staining of BAT sections. Scale bars: $100 \mu \mathrm{m}$. (I) BAT mRNA levels of thermogenic markers. Results are expressed as the mean \pm SEM. $n=5-6 \mathrm{KI}^{-/-}$and $n=9-10 \mathrm{WT}$ male mice on HFD per group. ${ }^{*} P<$ 0.05 versus WT determined by unpaired $t$ test. In $\mathbf{A}$ and $\mathbf{D}$, SEM and significance are not represented to enhance readability. Dark horizontal bars represent 12-hour dark phases.

similar to that of the WT mice (Figure 5A). The loss of DIO resistance in $\mathrm{Klb}^{-1-} \mathrm{Tgr5^{-/- }}$ mice is also evidenced by their similar fat mass and fat proportion compared with WT mice after HFD consumption (Figure 5, B and C). eWAT/BW ratio, $\mathrm{VO}_{2}$, and RER were not different between $\mathrm{Klb}^{-1-} \mathrm{Tgr} 5^{-1-}$ and WT mice (Figure 5, D-G). The $\mathrm{Kl}^{-1-} \mathrm{Tgr}^{-/}$mice were also similar to WT in terms of BAT weight, morphology, and gene expression (Figure 5, H-J), consistent with the loss of higher thermogenesis observed in $\mathrm{Klb}^{-1-}$ mice. Together, these data demonstrate that TGR5 signaling is involved in the increased energy expenditure and resistance to DIO in $\mathrm{Klb}^{-/-}$mice most likely mediating a modified BA signaling.

$\mathrm{Klb}^{-1-}$ mice have modified microbiota composition. BAs are well known to regulate gut microbiota, and in turn gut bacteria modulate the composition and the level of BAs (20-22). Thus, the large excess of the secondary BA DCA observed in $\mathrm{Kl}^{-/}$mice led us to assess their microbiota profile on both chow and HFD, using next-generation sequencing (NGS). Independently of the diet consumed, $\mathrm{Klb}^{-/-}$mice present a lower Shannon diversity index compared with WT (Figure 6A), reflecting more homogeneous populations of bacteria in their gut. Principal component analysis (PCA) based on the UniFrac distance (Figure 6B) shows different clustering positions of the $\mathrm{Klb}^{-1-}$ and WT mice within and across diets, indicating an interaction between diet and genotype. At the taxonomic level, we also identified a clear pattern of sample clustering by diet and genotype (Figure 6C). Analysis of microbiota composition at the phylum level indicates a moderate increase of the Proteobacteria proportion and a moderate decrease of the Firmicutes proportion in $\mathrm{Klb}^{-/-}$versus WT mice on chow diet (Figure 6, D and E). HFD consumption increased the Firmicutes proportion at the expense of the Bacteroidetes proportion in WT mice (Figure 6, D and E), as previously reported (23-25). Interestingly, HFD-fed $\mathrm{Klb}^{-1-}$ mice present a Bacteroidetes/Firmicutes ratio similar to WT mice on chow diet (Figure 6E, insert panel). At the class level, qualitative and quantitative changes in Bacteroidia, Clostridia, and Deltaproteobacteria explain the changes observed at the phylum level (data not 


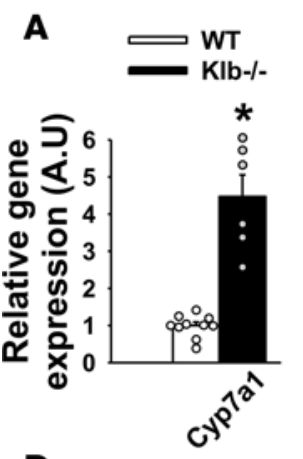

D
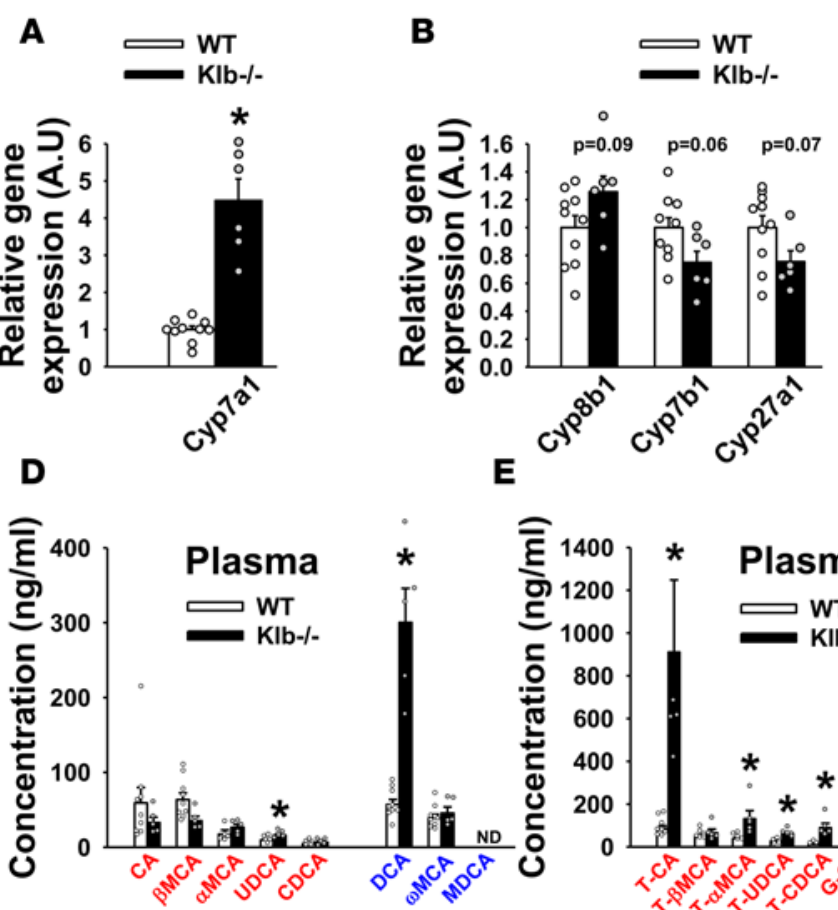

Primary BA Secondary BA

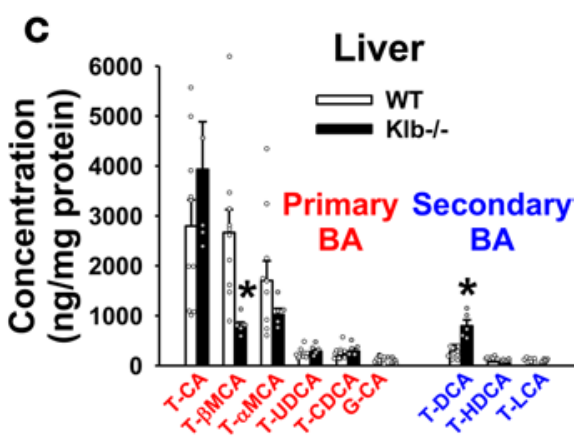

$\mathbf{F}$

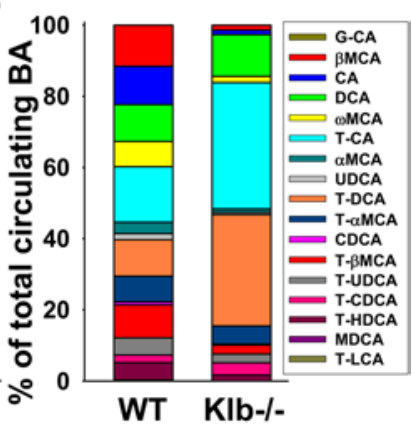

Figure 3. Modified circulating bile acid (BA) pool in $\mathbf{K I}^{-/-}$mice on high-fat diet (HFD). (A) Hepatic mRNA levels of cholesterol $7 \alpha$-hydroxylase enzyme. (B) Hepatic mRNA levels of enzymes in the classical and alternative BA synthesis pathways. (C) Hepatic levels of conjugated BAs. (D) Circulating levels of unconjugated BAs. (E) Circulating levels of conjugated BAs. (F) Circulating proportion of unconjugated and conjugated BAs. Results are expressed as the mean \pm SEM. $n=5-6 \mathrm{KIb}^{-/-}$and $n=9-10 \mathrm{WT}$ male mice on HFD per group. ${ }^{*} P<0.05$ versus WT determined by unpaired $t$ test. MCA, muricholic acid; CA, cholic acid; CDCA, chenodeoxycholic acid; DCA, deoxycholic acid; G-, glyco; HDCA, hyodeoxycholic acid; LCA, lithocholic acid; MDCA, murocholic acid; ND, not determined; T-, tauro-; UDCA, ursodeoxycholic acid. Individual values not represented (outside the range of the $y$ axis): C (7,422 for T-CA in KI $b^{-/-}$group) and $\mathbf{E}$ (2,248 for T-CA in $\mathrm{KI}^{-/-}$group).

shown). We then analyzed the corresponding bacterial families and genera in more detail. At the family level, HFD-fed $\mathrm{Klb}^{-/-}$mice display a decreased proportion of Lachnospiraceae and Clostridiales vadinBB60 and an increased proportion of Prevotellaceae and Porphyromonadaceae, and a trend towards an increase proportion of Bacteroidaceae and Bacteroidales S24-7 group compared with HFD-fed WT mice (Figure $6 \mathrm{~F})$. At the genus level, $\mathrm{Klb}^{-1-}$ mice on HFD show an increased proportion of Parabacteroides, Bacteroides, Rikenella, Alloprevotella, Eubacterium coprostanoligenes, and Akkermansia, and a reduced proportion of Oscillibacter, Ruminococcus, and genera within Lachnospiraceae compared with HFD-fed WT mice (Figure 6G). Globally, these data demonstrate that the classical change in gut microbiota pattern induced by HFD consumption was mitigated in $\mathrm{Klb}^{-1-}$ mice.

Resistance of $\mathrm{Klb}^{-1-}$ mice to DIO is DCA driven and microbiota dependent. The massive increase of DCA and T-DCA levels in $\mathrm{Klb}^{-1-}$ mice led us to investigate the specific contribution of this secondary BA to the increase in energy expenditure on HFD. To this end, we treated orally $(0.5 \mathrm{~g} / 1$ in drinking water) both $\mathrm{Klb}^{-/-}$and WT mice with vancomycin (VCM) 1 week before initiation and during the entire period of HFD consumption. VCM is a poorly absorbed antibiotic preferentially targeting Gram-positive bacteria, including Clostridium species classically described as responsible for the conversion of primary BAs into secondary BAs (such as $7 \alpha$-dehydroxylation of CA into DCA; see ref. 26). VCM treatment similarly reshapes the gut microbiota of $\mathrm{Klb}^{-1-}$ and WT mice, massively decreasing both intestinal Bacteroidetes and Firmicutes content in both genotypes on HFD (Figure 7A). In contrast, Proteobacteria content was not impacted by VCM in WT and showed an increasing trend in $\mathrm{Klb}^{-/-}$mice (Figure 7A). Notably, the reshaped microbiota presents drastically reduced $7 \alpha$-dehydroxylation activity, leading to residual circulating levels of DCA and T-DCA (Figure 7, B and C). Instead, the circulating BA pool is mainly composed of T- $\beta$ MCA and T-CA in WT mice and of T-CA in $\mathrm{Klb}^{-1-}$ mice on HFD+VCM (Figure 7D). The specific pattern of primary BA synthesis due to $K l b$ deficiency was not affected by VCM treatment, since HFD-fed $\mathrm{Klb}^{-1-}$ mice presented again 
A

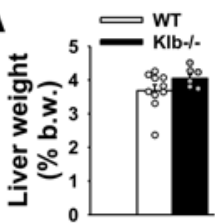

B
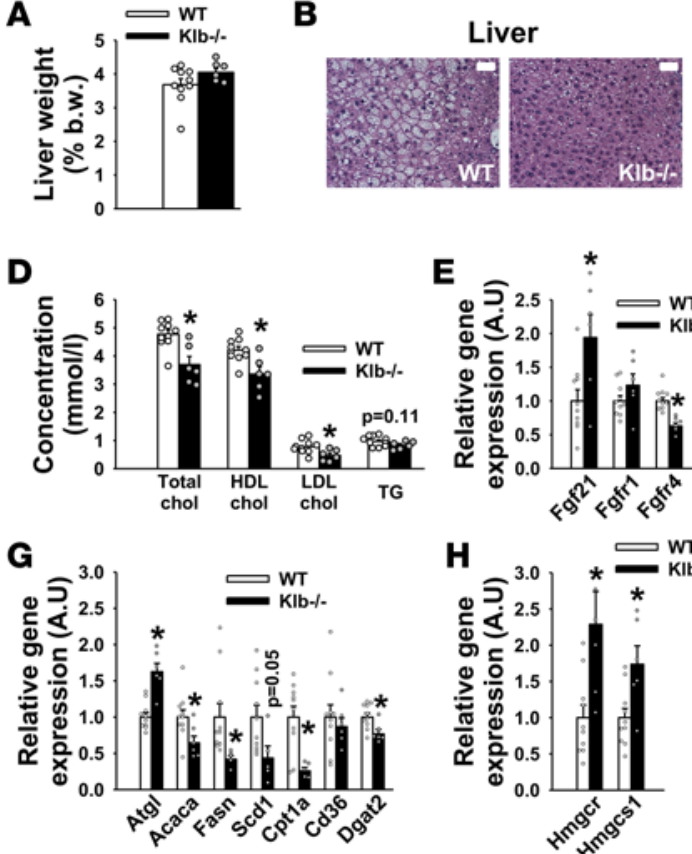

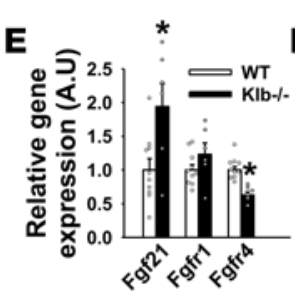

H

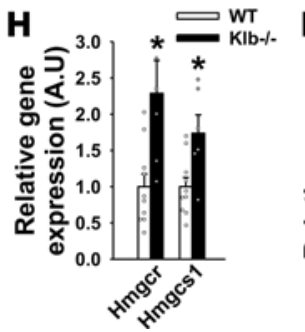

I

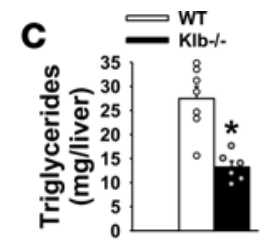

WT
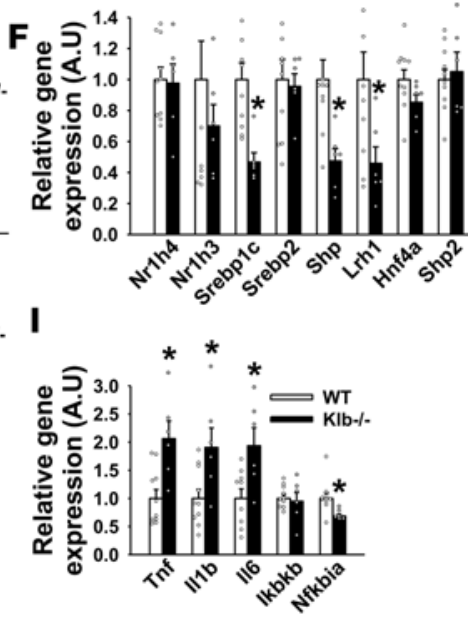

Figure 4. Protection against fatty liver in $\mathrm{KI}^{-/-}$mice on high-fat diet (HFD). (A) Liver weight. (B) H\&E staining of liver sections. Scale bars: $100 \mu \mathrm{m}$. (C) Hepatic triglyceride content. (D) Circulating total-, HDL-, and LDLcholesterol levels and triglyceride levels. (E) Hepatic mRNA levels of ligand and receptors of the FGF signaling pathway. (F) Hepatic mRNA levels of transcriptional regulators. (G) Hepatic mRNA levels of metabolic enzymes and transporters. (H) Hepatic mRNA levels of cholesterogenesis enzymes. (I) Hepatic mRNA levels of inflammatory markers.

Results are expressed as the mean \pm SEM. $n=$ 5-6 $\mathrm{Klb}^{-1-}$ and $n=9-10 \mathrm{WT}$ male mice on HFD per group. ${ }^{*} P<0.05$ versus WT determined by unpaired $t$ test. Individual values not represented (outside the range of the $y$ axis): $\mathbf{F}(1.88,1.44,1.80,2.35$ for Nr1h3 in WT group; 1.60 for Srebp1c in WT group; 1.76 for Srebp2 in WT group; 1.79, 1.56 for Shp in WT group; 1.97, 1.59 for Lrh1 in WT group) and $\mathbf{H}$ (4.16 for Hmger in $\mathrm{KIb}^{-/-}$group).

higher levels of Cyp7a1 and Cyp8b1 and lower levels of Cyp7b1 compared with HFD-fed WT mice (Supplemental Figure 2A). Importantly, following VCM administration, $\mathrm{Klb}^{-/-}$mice lose their resistance to DIO. Indeed, they gain similar weight (Figure 7E) and exhibit similar fat proportion and content to that of WT (Figure 7F and Supplemental Figure 2B), despite a still lighter eWAT (Figure 7G); no major change was observed in adipose-targeted gene expression (Supplemental Figure 2, C and D), glucose tolerance (Figure $7 \mathrm{H}$ ), or stool energy content (Figure 7I). Consistently, BAT weight (Figure 8A) and morphology (Figure 8B) were indistinguishable between $\mathrm{Klb}^{-/-}$and WT mice on HFD+VCM. VO (Figure 8, C and D), $\mathrm{VCO}_{2}$ (Supplemental Figure 2, E and F), and RER (Figure 8E) were also similar between $\mathrm{Klb}^{-/-}$and WT mice on HFD+VCM. Gene expression analysis in this tissue confirmed the abrogation of thermogenesis induction in $\mathrm{Klb}^{-/-}$mice on HFD+VCM, with a trend towards lower Ucp1, Dio2, and Elovl3 expression levels and significantly lower Pgcla and Cidea expression levels (Figure 8F). We also observed a massive increase in liver weight (Figure $8 \mathrm{G}$ ), indicating a preferential storage of fat in the liver rather than in the WAT of $\mathrm{Klb}^{-{ }^{--}}$mice in the absence of DCA-driven BAT overactivity (Figure 8, H and I). This observation is also corroborated by increased hepatic expression of the scavenger receptor (fatty acid translocase, $C d 36$ ) involved in lipid accumulation under excessive fat supply (Figure 8J). Finally, hepatic gene expression of cholesterogenesis enzymes (Figure $8 \mathrm{~K}$ ) and proinflammatory cytokines (Figure $8 \mathrm{~L}$ ) remained elevated in $\mathrm{Klb}^{-/-}$mice on HFD+VCM.

\section{Discussion}

FGF21 is one of the most promising drug candidates for treating obesity and type 2 diabetes (4); this is based on the metabolic protection observed in pharmacological administration studies (5), as well as in murine studies of transgenic overexpression $(5,6)$. In contrast, some metabolic abnormalities have been reported in $F g f 1^{-1-}$ mice. This includes impaired lipid utilization $(27,28)$, defects in gluconeogenesis and ketogenesis $(29,30)$, and defects in WAT browning (7) and BAT glucose uptake (31). Together, these results raise questions about the function of endogenous FGF21. $\beta$-Klotho, the obligate coreceptor for FGF21/FGFR1c signaling $(2,3)$, has been targeted to selectively abrogate FGF21 action in vivo $(9,10)$. In particular, tissueselective $\beta$-klotho deletions in adipose tissue (10) and in brain (11-13) have revealed the site of action of pharmacologic FGF21. FGF21 acts simultaneously on adipocytes and the hypothalamus to provide both the oxidative substrate and the central sympathetic outflow to drive thermogenesis and weight loss (4).

Thus, we expected that whole-body $\mathrm{Klb}^{-/-}$mice would be prone to developing metabolic disorders under hypercaloric feeding conditions (i.e., HFD) in the absence of endogenous FGF21 signaling. Counterintuitively, we observed that these mice are resistant to obesity due to an elevation in energy 
A

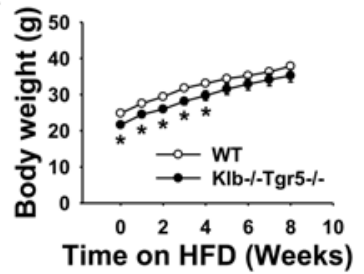

E

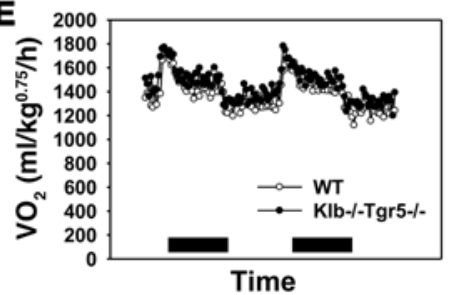

B

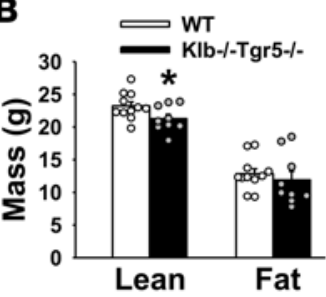

$\mathbf{F}$

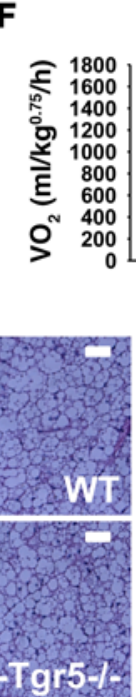

I

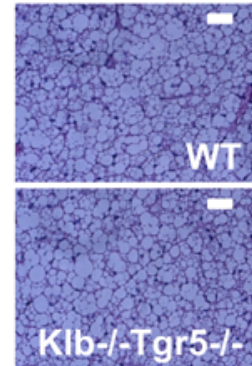

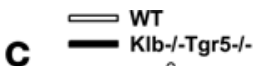
$\circ \circ$
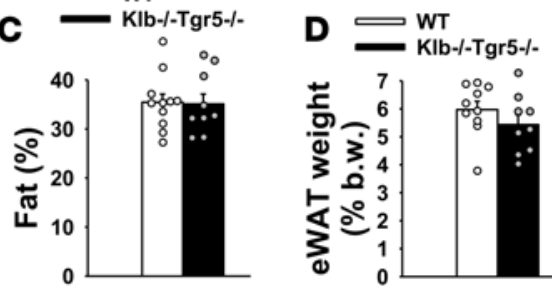

G
H

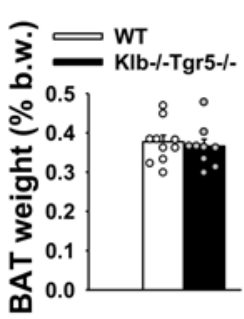

\section{WT}

KIb-/Tgr5- $/$.

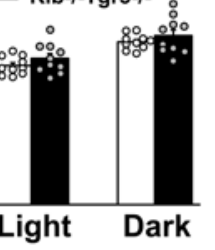

\section{0}

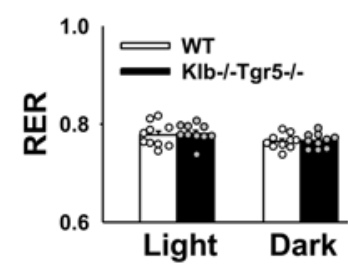

Light Dark

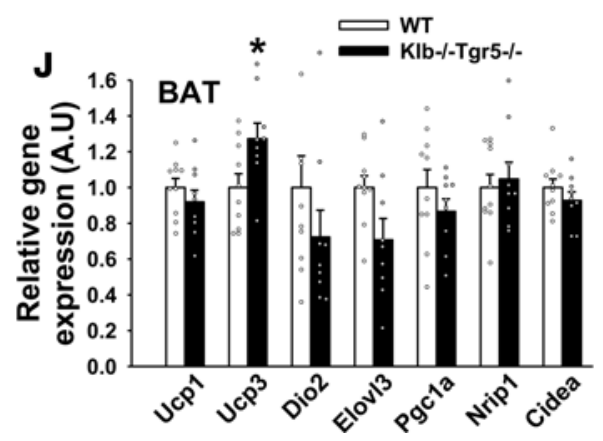

Figure 5. Concomitant genetic deletion of Tgr5 abrogates resistance to high-fat diet-induced obesity in $\mathrm{KIb}^{-/-}$mice. (A) Body weight curve. HFD, high-fat diet. (B) Lean and fat mass. (C) Fat proportion. (D) Epididymal white adipose tissue (eWAT) weight. (E) $\mathrm{O}_{2}$ consumption $\left(\mathrm{VO}_{2}\right.$ ) curves representing 2 consecutive days. Dark horizontal bars represent 12-hour dark phases. (F) Mean $\mathrm{VO}_{2}$ during light and dark phases. (C) Respiratory exchange ratio $\left(\mathrm{RER}=\mathrm{VCO}_{2} / \mathrm{VO}_{2}\right.$, where $\mathrm{VCO}_{2}$ is $\mathrm{CO}_{2}$ production) during light and dark phases. (H) Brown adipose tissue (BAT) weight. (I) H\&E staining of BAT sections. Scale bars: $100 \mu \mathrm{m}$. (J) BAT mRNA levels of thermogenic markers. Results are expressed as the mean \pm SEM. $n=9-10 \mathrm{Klb}^{-/-} \operatorname{Tgr5}^{-/-}$and $n=10-11$ WT male mice on HFD per group. ${ }^{*} P<0.05$ versus WT determined by unpaired $t$ test.

expenditure associated with BAT overactivity. In addition to the interesting therapeutic value of pharmacological recombinant FGF21 or FGF21 analogs, this surprising result leads to questions about the role of endogenous FGF21 signaling in adaptation to hypercaloric diet and suggests the existence of compensatory mechanisms underlying this metabolic protection.

$\beta$-Klotho is also the obligate coreceptor for FGF(15/19)/FGFR4 signaling occurring in the liver (14, 15). Similar to the $\mathrm{Klb}^{-/-}$mice, $\mathrm{Fgfr}^{-/-}$mice also exhibit improved metabolic status on HFD (32). However, the supraphysiologic levels of FGF15 and FGF21 observed in $\mathrm{Fgfr}^{-1-}$ mice might alleviate the consequences of HFD consumption by overstimulating the intact FGFR1c/ $\beta$-klotho complex. In contrast, the $\mathrm{Klb}^{-/-}$mice have concomitant deficiencies in both FGF15/FGFR4 and FGF21/FGFR1c signaling pathways. Initial characterization revealed that the $\mathrm{Klb}^{-1-}$ mice have a derepression of BA production and are resistant to diet-induced gallstone formation (17). We now demonstrate that global $\beta$-klotho deficiency elevated thermogenesis and BAT activity on HFD, underlying a resistance to obesity. Our results are in accordance with a recent report showing limited weight gain in $\mathrm{Klb}^{-1-}$ mice on HFD without mechanistic explanation of the observed phenomenon (18).

We presently show that $\mathrm{Klb}^{-/-}$mice exhibit a drastic shift in primary BA production with activation of the classical (neutral) BA pathway (higher hepatic Cyp861 expression and T-CA levels) at the expense of the alternative (acidic) BA pathway (lower hepatic Cyp7b1 expression and T- $\beta$ MCA levels). This reveals that the ileo-hepatic FGF15/FGFR4/B-klotho negative feedback not only regulates the rate of BA synthesis but also alters BA composition in mice, as the hepatic FXR/MAFG pathway (33). Genetic and pharmacological approaches allowed us to demonstrate that the modified BA homeostasis is directly involved in activating 
A

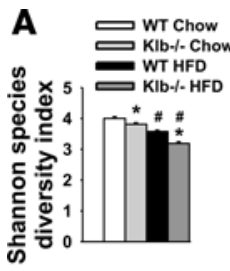

B

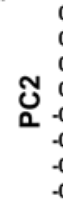

0.3
0.2
0.1
บु 0.0
-0.1
-0.2
-0.3
-0.4

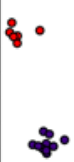

.

D

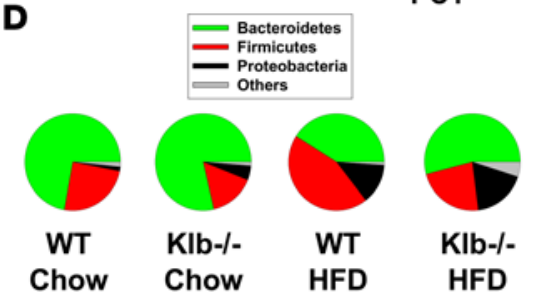

E

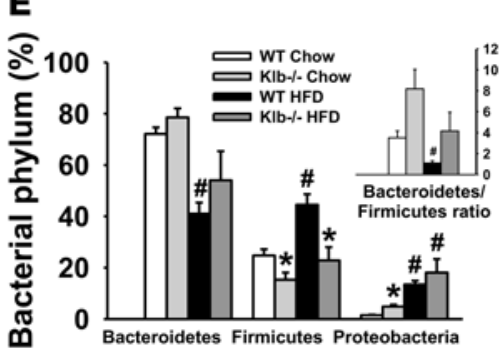

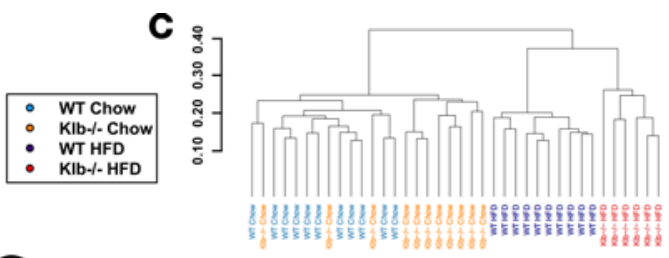

0.3

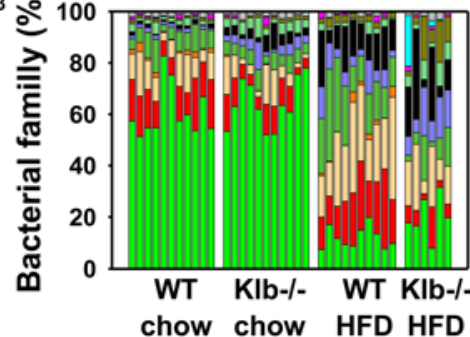

G

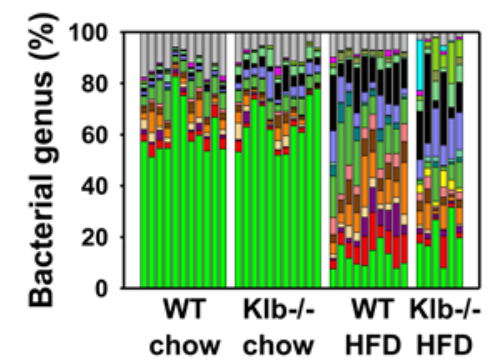

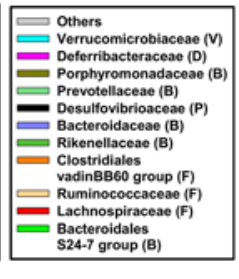

OAkers - Mucispirillum (D) Parabacteroides (B) Alloprevotella (B) - Desulfovibrio (P) - Rikenellaceae Alistipes (B) coprostanoligenes group (F) Anrestanoligenes Ruminiclostridium 9 - Ruminococcaceas Oscillibacter Oscillibacter
Blautia (F)
Lachnospirace uncultured (F)
Bacteroidales Bacteroidales
S24-7 group (B)

Figure 6. Modification of gut microbiota composition in $\mathbf{K I}^{-/-}$mice on chow and high-fat diet (HFD). (A) Shannon species diversity index. (B) Principal component analysis (PCA). (C) Dendrogram of UniFrac distances illustrating the phylogenetic clustering. (D) Proportions of gut bacteria at the phylum level. (E) Relative abundance of gut bacteria at the phylum level. (F) Relative abundance of gut bacteria at the family level. (G) Relative abundance of gut bacteria at the genus level. In $\mathbf{A}$ and $\mathbf{E}$, results are expressed as the mean \pm SEM. Chow diet: $n=11 \mathrm{KI}^{-/-}$and $n=11 \mathrm{WT}$ male mice. HFD: $n=6 \mathrm{KIb}^{-/-}$and $n=10$ WT male mice. ${ }^{*} P<0.05$ versus diet-matched WT (genotype effect); ${ }^{*} P<0.05$ for HFD versus chow group for the same genotype (diet effect) determined by ANOVA. In F and G, each column represents the microbiota of 1 animal. B, Bacteroidetes; F, Firmicutes; P, Proteobacteria; D, Deferribacteres; V, Verrucomicrobia.

thermogenesis in HFD-fed $\mathrm{Klb}^{-/-}$mice. Indeed, the double-KO mice lacking both $\mathrm{Klb}$ and the membrane BA receptor $\operatorname{Tgr} 5\left(\mathrm{Klb}^{-/-} \mathrm{Tgr} 5^{-/}\right)$are similar to WT in terms of fat accretion and energy expenditure, reflecting that these mice lose their protection against DIO. These results are in line with previous works demonstrating that obesity can be prevented in mice receiving either nutritional supplementation with CA (19) or BA-binding resin (34). Despite opposite effects on BA synthesis, both of these interventions modify the BA composition, enriching the BA pool in CA and derivatives. Mechanistically, the activation of TGR5 in the BAT by hydrophobic BA led to a local Dio2 overexpression, thus driving tissular T3 elevation and stimulating thermogenesis (19). Together, these results confirm that BA composition, in particular a high hydrophobicity, is a key endocrine signal driving thermogenesis in mice during nutritional fat overload.

A striking finding is our observation that the secondary BA DCA (in its conjugated and unconjugated forms) is the most abundant BA species in the circulation of $\mathrm{Klb}^{-1-}$ mice. Generating DCA requires deconjugation of T-CA and subsequent $7 \alpha$-dehydroxylation of CA into DCA. Both steps are catalyzed by bacterial enzymes (26). To dissociate the thermogenic potency of the primary CA and its secondary derivative DCA in vivo, we treated HFD-fed $\mathrm{Klb}^{-1-}$ mice with the antibiotic VCM. This induced a massive shift in microbiota composition featured by a dramatic reduction in Gram-positive Bacteroidetes and Firmicutes. VCM treatment drastically abates conversion of CA into DCA, resulting in high CA levels and residual DCA levels (at the limit of detection). In this setting, the $\mathrm{Klb}^{-/-}$mice also lost their resistance to DIO, highlighting DCA as the main driver of thermogenesis and BAT stimulation. The specific thermogenic property of DCA presently observed in vivo is consistent with its higher potency to activate TGR5 in vitro (35). These observations add another layer of complexity to the thermogenic properties of BA, as gut microbiota appear to be a key intermediary in this process. Growing attention has been recently given to 

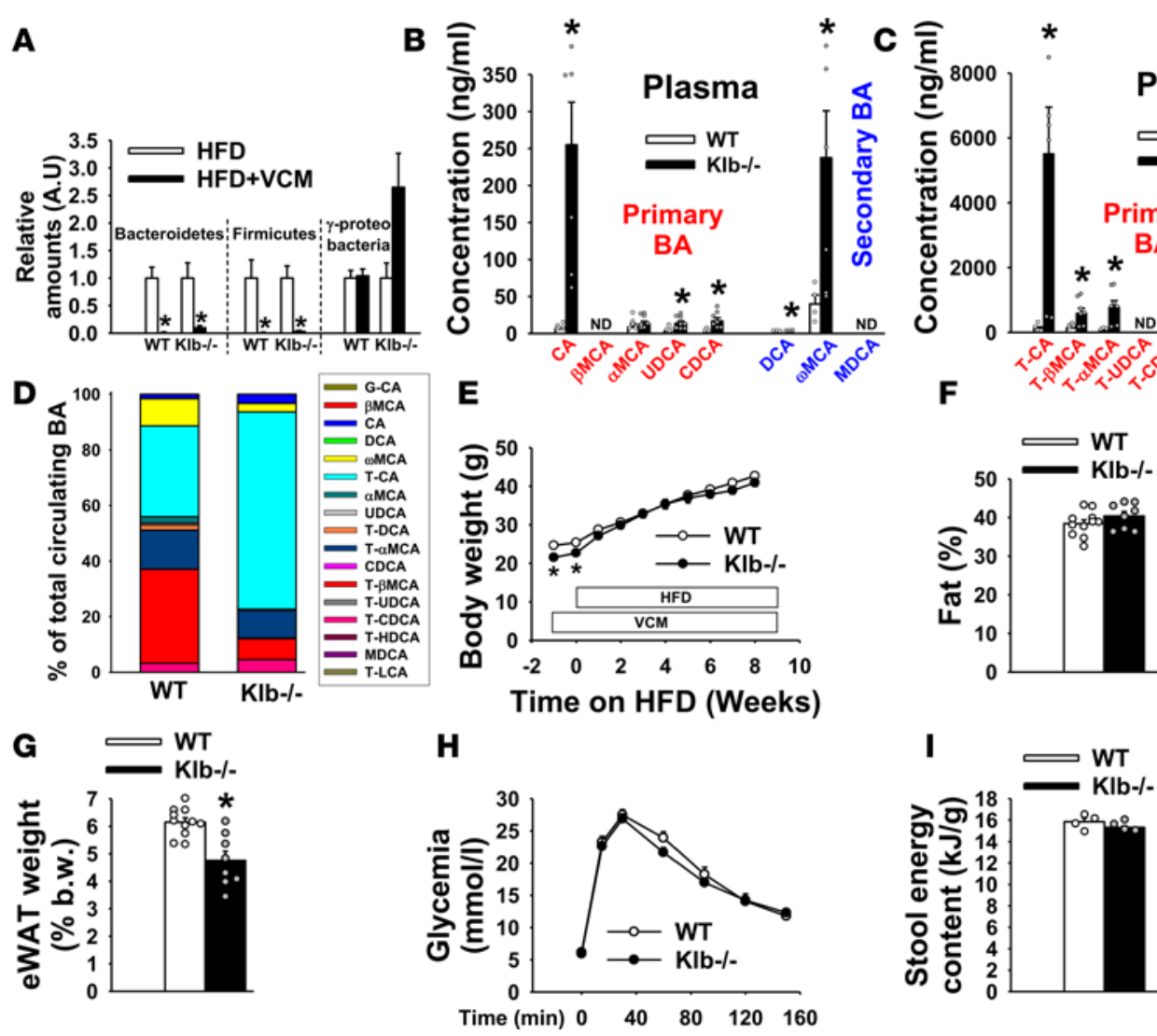

Figure 7. Antibiotic-mediated blockade of deoxycholic acid (DCA) production abrogates resistance to high-fat diet-induced obesity in $K^{-/ /-}$mice. (A) Relative abundance of gut bacteria at the phylum/class level. HFD, high-fat diet; VCM, vancomycin. (B) Circulating levels of unconjugated bile acids (BAs). (C) Circulating levels of conjugated BAs. (D) Circulating proportion of unconjugated and conjugated BAs. (E) Body weight curve. (F) Fat proportion. (C) Epididymal white adipose tissue (eWAT) weight. (H) Glucose tolerance test. (I) Stool energy content. Results are expressed as the mean \pm SEM. A-D (HFD+VCM): $n$ $=6 \mathrm{KIb}^{-/-}$and $n=6$ WT male mice per group. E-I (HFD+VCM): $n=8 \mathrm{KI} / \mathrm{b}^{-/-}$and $n=11 \mathrm{WT}$ male mice per group. For I, each individual value represents a pool of 2 to 3 mice. ${ }^{*} P<0.05$ versus WT determined by unpaired $t$ test. Individual values not represented (outside the range of the $y$ axis): B (414 for CA and 445 for $\omega \mathrm{MCA}$ in $\mathrm{KIb}^{-/-}$group) and C (10,594 for T-CA in $\mathrm{KI}^{-/-}$group). MCA, muricholic acid; CA, cholic acid; CDCA, chenodeoxycholic acid; DCA, deoxycholic acid; G-, glyco; HDCA, hyodeoxycholic acid; LCA, lithocholic acid; MDCA, murocholic acid; ND, not determined; T-, tauro-; UDCA, ursodeoxycholic acid.

the metabolic role of microbiota-dependent BA transformation (36-38). Most recent advances have shown that the level of BA deconjugation functionally regulates host lipid metabolism (39) and that gut microbiota could promote DIO through modulation of the BA profile, impacting FXR signaling (40, 41). This concept also covers human physiology; short-term oral administration of VCM to obese subjects concomitantly decreased secondary BA levels and lowered peripheral insulin sensitivity (42). It should be noted that the thermogenic properties of DCA specifically involve the stimulation of BAT, because $\mathrm{Klb}^{-1-}$ mice showed no sign of browning in different WAT depots (epididymal, perirenal, and inguinal); this may stem from the low Tgr5 expression levels in these fat pads or absence of the cellular machinery needed for DCA signaling.

Several recent insights have also contributed to our understanding of the complex BA-mediated crosstalk between the liver and the gut microbiota. On one hand, the detergent properties of BAs exert a selective pressure on the intestinal bacteria; this regulates the proliferation and composition of the gut microbiota (20). In turn, gut microbiota modulate both the composition of the BA pool (through chemical transformations) and its amount (through modulation of the ileo-hepatic FXR/FGF15/FGFR4/ $\beta$ klotho axis that represses BA production; see refs. 21, 22). DCA is both generated by bacteria and has the highest antibacterial capacity among the BAs (20). The elevated DCA levels observed in $K l b^{-1-}$ mice raises questions about their gut microbiota composition. In basal conditions (chow diet), the microbiota content of $\mathrm{Klb}^{-/-}$mice shows few modifications compared with WT. Interestingly, these moderate changes were largely amplified on HFD. In WT animals, fat intake is well known to specifically enrich gut microbiota in Firmicutes at the expense of Bacteroidetes (23-25). In contrast, the $\mathrm{Klb}^{-/-}$mice exhibited a markedly higher Bacteroidetes/Firmicutes ratio on HFD compared with WT. Since Firmicutes-enriched 


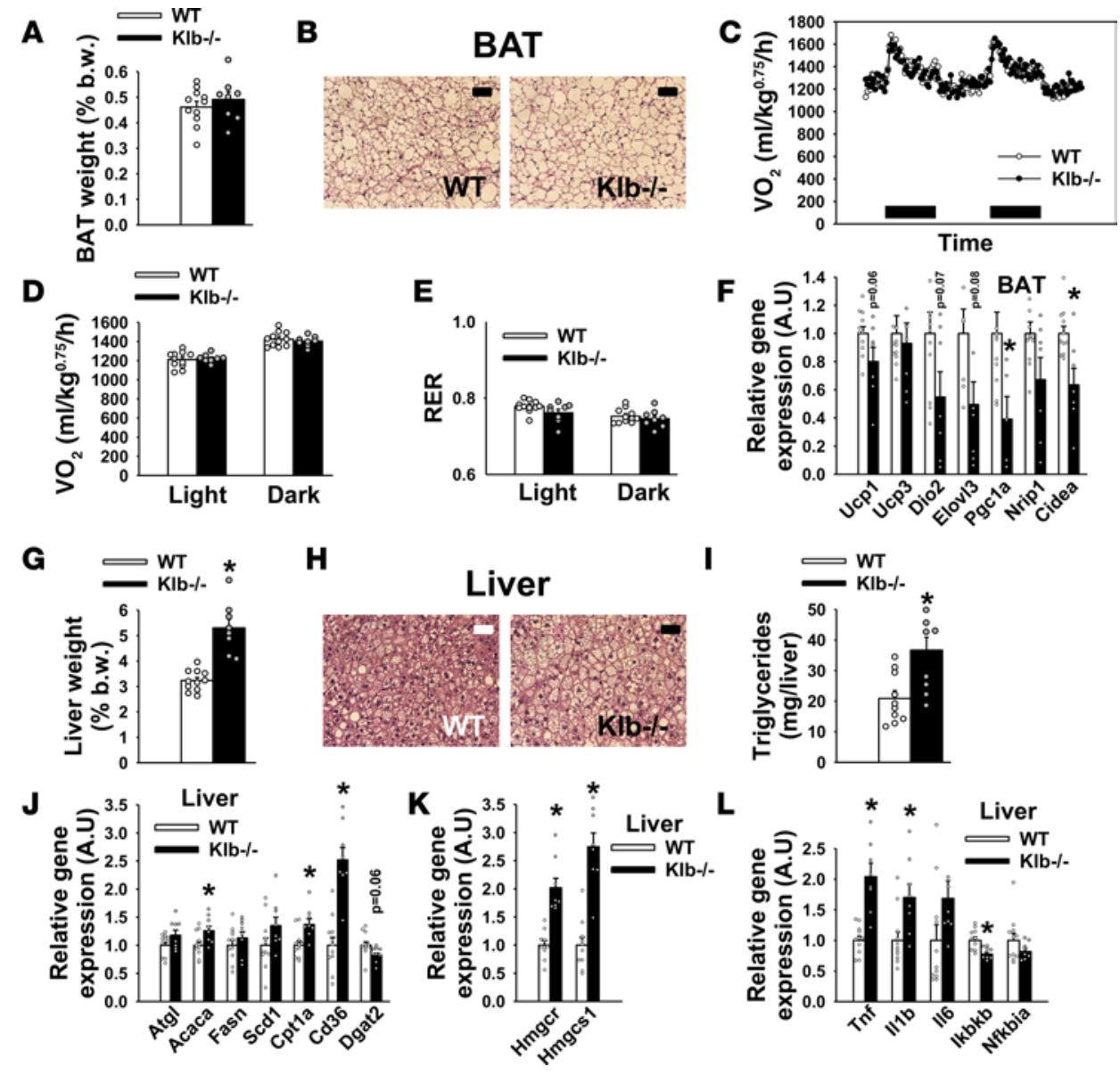

Figure 8. Antibiotic-mediated blockade of deoxycholic acid (DCA) production abrogates BAT activation and fatty liver protection in $\mathrm{KI}^{-/-}$mice on high-fat diet (HFD). (A) Brown adipose tissue (BAT) weight. (B) H\&E staining of BAT sections. (C) $\mathrm{O}_{2}$ consumption $\left(\mathrm{VO}_{2}\right)$ curves representing 2 consecutive days. Dark horizontal bars represent 12-hour dark phases. (D) Mean $\mathrm{VO}_{2}$ during light and dark phases. (E) Respiratory exchange ratio ( $\mathrm{RER}=\mathrm{VCO}_{2} / \mathrm{VO}_{2}$, where $\mathrm{VCO}_{2}$ is $\mathrm{CO}_{2}$ production) during light and dark phases. (F) BAT mRNA levels of thermogenic markers. (C) Liver weight. (H) H\&E staining of liver sections. (I) Hepatic triglyceride content. (J) Hepatic mRNA levels of metabolic enzymes and transporters. (K) Hepatic mRNA levels of cholesterogenesis enzymes. (L) Hepatic mRNA levels of inflammatory markers. Results are expressed as mean \pm SEM. $n=8$ $K I b^{-1-}$ and $n=11 \mathrm{WT}$ male mice on HFD+VCM per group. ${ }^{*} P<0.05$ versus WT determined by unpaired $t$ test. Scale bars: $100 \mu \mathrm{m}$ (B and $\mathbf{H}$ ). Individual values not represented (outside the range of the $y$ axis): $\mathbf{F}$ (2.21 for Ucp3, 2.18 for Dio2, 1.78 for Elolv3, 1.70 and 2.14 for Pgc1a, and 1.61 for Nrip in WT group; 1.58 for Ucp3 and 1.57 for Elolv3 in $\mathrm{KI}^{-/-}$group) and $\mathbf{L}$ (3.49 for 116 in $\mathrm{KI}^{-/-}$group). microbiota show enhanced energy harvesting from food (25), the relative underrepresentation of Firmicutes in $\mathrm{Klb}^{-/-}$mice could attenuate energy assimilation and potentially contributes to the observed resistance to DIO. However, we saw a trend towards decreased fecal caloric content in HFD-fed $\mathrm{Klb}^{-/-}$compared with HFD-fed WT mice, suggesting at the very least equivalent energy harvesting efficiency. These results suggest that the specific microbiota composition in HFD-fed $\mathrm{Klb}^{-/-}$mice is not the primary cause of their resistance to DIO. Nevertheless, further studies are warranted to elucidate whether the specific microbiota observed in $\mathrm{Klb}^{-/-}$mice can impact host energy partitioning via changes in short-chain fatty acid production $(43,44)$ or intestinal BA signaling (41, 45, 46).

Hepatosteatosis (nonalcoholic fatty liver, NAFL) is a classic complication of obesity. Both endocrine FGFs and BAs are involved in regulating hepatic lipid metabolism (47, 48). Previous murine studies have demonstrated that genetic ablation or adenoviral knockdown of Fgf21 promotes hepatic fat accumulation $(27,49)$. The underlying mechanism could involve enhanced SREBP1c maturation (27) and/or increased ER stress in hepatocytes (50). In contrast, we observed that HFD-fed $\mathrm{Klb}^{-/-}$mice are protected against hepatosteatosis, as was previously shown for HFD-

fed $\mathrm{Fg}_{\mathrm{fr}} \mathrm{4}^{-1-}$ mice (51). These data suggest that disruption of hepatic FGF15/FGFR4/ $\beta$-klotho signaling overcomes FGF21/FGFR1c/ $\beta$-klotho signaling deficiency, preventing hepatic fat storage on HFD. The high BA levels seen in both $\mathrm{Klb}^{-/-}$and $\mathrm{Fgfr}^{-/-}$models might mediate this protection against hepatic fat storage. Cyp7a1-transgenic mice with elevated BA levels are also resistant to hepatosteatosis (52). Srebp1c is a key lipogenic transcription factor and is downregulated by CA or FXR agonists both in vivo and in vitro (53). Interestingly, HFD-fed $\mathrm{Klb}^{-/-}$mice have low hepatic expression levels of Srebp1c (and its target enzymes Acaca and Fasn) compared with HFD-fed WT mice, reflecting a BA-mediated repression of de novo hepatic lipogenesis. Further gene expression analyses in HFD-fed $\mathrm{Klb}^{-1-}$ mice also revealed decreased hepatic triglyceride synthesis and increased triglyceride breakdown, in line with disrupted hepatic fatty acid availability. Interestingly, VCM administration to HFD-fed $\mathrm{Klb}^{-/-}$mice abolished not only resistance to DIO but also resistance to hepatosteatosis. This latter effect should also result from modified BA composition. Indeed, the abrogation of DCA production both mitigates the repression of de novo hepatic lipogenesis (DCA being a strong FXR agonist) and indirectly leads to hepatic fatty acid uptake (reflected in Cd36 upregulation) in the setting of decreased BAT lipid utilization. Consequently, the $\mathrm{Klb}^{-/-}$mice on HFD+VCM exhibit altered body fat distribution with a massively enlarged fatty liver (nearly double that 

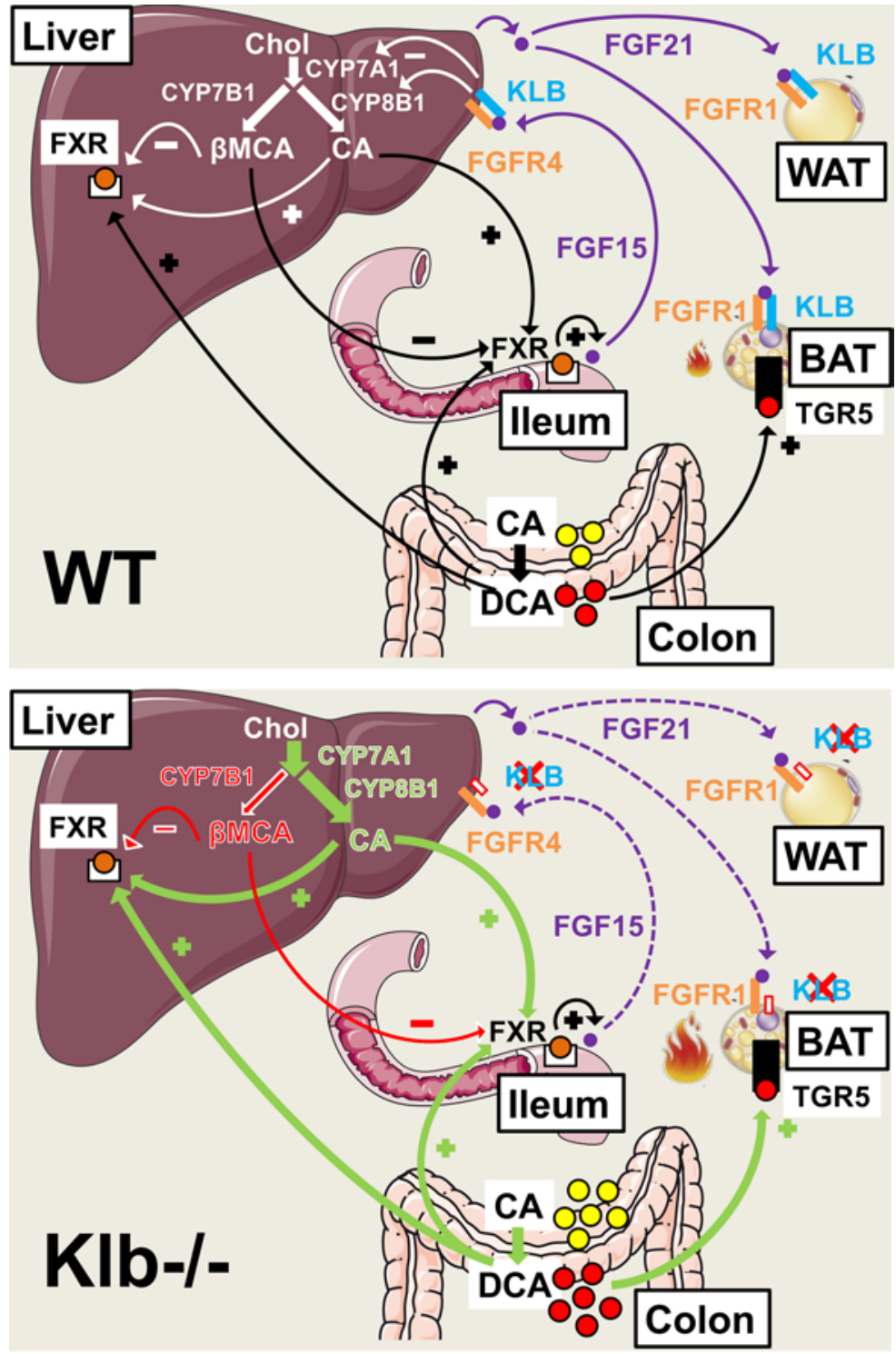

Figure 9. Model of balance between endocrine FGF and bile acid (BA) signaling in WT and $\mathrm{KI}^{-/-}$mice on high-fat diet (HFD). $\beta$-Klotho deficiency leads to a derepressed (elevated) BA synthesis and major changes in BA composition. This features an upregulation of the classic BA synthesis pathway (Cyp8b1) at the expense of the alternative BA synthesis pathway (Cyp7b1). The result is an excess of cholic acid (CA) leading to a large overproduction of microbiota-derived deoxycholic acid (DCA). DCA specifically activates thermogenesis in the brown adipose tissue (BAT) through the membrane receptor TCR5 and confers resistance to diet-induced obesity even in the absence of endogenous FGF21 signaling. Upregulation and/or stimulation are represented by green arrows. Downregulation and/or inhibition are represented by red arrows. $\beta M C A, \beta$-muricholic acid; Chol, cholesterol; WAT, white adipose tissue.

of WT). Thus, in the absence of DCA-driven BAT thermogenesis, $\mathrm{Klb}^{-/}$mice become susceptible to hepatosteatosis and phenocopy $\mathrm{Fg}_{2} 21^{-/-}$mice $(27,49)$.

Compared with WT, $\mathrm{Klb}^{-1-}$ mice exhibit increase levels of inflammation markers. This constitutive hepatic proinflammatory state is independent of hepatic fat storage or amount of DCA, unlikely reflecting a deleterious consequence of BA excess. Moreover, Cyp7a1-transgenic mice show reduced hepatic inflammation despite high BA levels (52). Thus, the hepatic inflammation observed in $\mathrm{Klb}^{-1}$ mice may result directly from the lack of FGF21 signaling. Several reports have recently described a feedback loop between FGF21 signaling and inflammation; inflammatory mediators decrease FGF21 signaling through $K l b$-specific repression $(54,55)$, and in turn, FGF21 signaling exerts antiinflammatory effects (56-58). Interestingly, in the nonobese model of fatty liver disease, FGF21 deficiency also worsened ER stress and inflammation $(59,60)$. Administration of FGF21 reversed these alterations, demonstrating a hepatoprotective action $(59,60)$.

To summarize, we document that $\mathrm{Klb}^{-1-}$ mice are resistant to DIO. The underlying mechanism involves activation of the classical (neutral) BA synthesis pathway at the expense of the alternative (acidic) pathway. High levels of hepatic CA production result in a large excess of microbiota-derived DCA. This secondary BA is specifically responsible for activating the TGR5 signaling pathway that stimulates BAT thermogenic activity (Figure 9). In the absence of DCA-driven thermogenesis, $\mathrm{Klb}^{-1-}$ mice recover susceptibility to DIO yet display altered body fat distribution including ectopic hepatic fat storage. These data demonstrate that gut microbiota can be a key regulator of host thermogenesis via secondary BA production. From a translational point of view, it should be considered that BA metabolism is quite different between mice and humans. In particular, the persistence of chenodeoxycholic acid (CDCA) (instead of the rapid conversion into MCA in rodents) modifies the relative hydrophobicity of other BA species and possibly their signaling properties through TGR5 and FXR. Nevertheless, the thermogenic activity of BA seems conserved in humans. Oral supplementation with CDCA increased BAT activity and energy expenditure in healthy women (61). Using a pharmacologic or nutritional approach to selectively modulate BA composition may be a promising target for treating metabolic disorders.

\section{Methods}

\section{Animal studies}

Models, housing, and breeding. We deeply thank Yo-ichi Nabeshima (Department of Pathology and Tumor Biology, Kyoto University School of Medicine, Kyoto, Japan) for providing the previously 
described $\mathrm{Klb}^{-/-}$mouse model (17). Neonatal overmortality of $\mathrm{Klb}^{-/-}$mice, previously reported on a mixed genetic background (17), was worsened on a pure C57BL/6J background, with only $5 \%$ to $10 \%$ of $\mathrm{Klb}^{-/-}$mice obtained from $\mathrm{Klb}^{+/-}$breeding. Double-KO $\mathrm{Klb}^{-1-} \mathrm{Tgr}^{-1-}$ mice were obtained through crossing $\mathrm{Klb}^{-/-}$(17) and $\mathrm{Tgr}^{-/-}$mice (62). All animals used in this study were males backcrossed onto a pure C57BL/6J background for more than 10 generations. After weaning, $\mathrm{Klb}^{-/-}, \mathrm{Klb}^{-/-} \mathrm{Tgr}^{-/-}$, and WT littermates were housed in standardized conditions $\left(22^{\circ} \mathrm{C}, 12\right.$-hour light/dark cycle, free access to water and food) until sacrifice.

Diets. Mice received a normal chow diet containing 4.5\% fat (diet 3436, Provimi Kliba) from weaning to 10 weeks of age, then switched to a HFD containing $60 \%$ of calories from fat (diet D12492, Research Diets) until sacrifice. BW was determined weekly.

Antibiotic treatment. One week prior to initiating HFD, mice received VCM (TEVA) via sterile drinking water at a concentration of $0.5 \mathrm{~g} / 1$. Water supply was renewed every 3 days until sacrifice.

Body composition. Lean mass, fat mass, and percentage fat were determined using MRI (Echo Medical Systems).

Glucose tolerance test. Glucose ( $2 \mathrm{mg} / \mathrm{g} \mathrm{BW}$ ) was injected intraperitoneally following an overnight fast.

Food intake. Food intake was measured for mice housed in individual cages for 3 consecutive days.

Indirect calorimetry and physical activity. After 48 hours of acclimatization, mean $\mathrm{VO}_{2}, \mathrm{VCO}_{2}$, and cumulative spontaneous locomotor activity were measured during 2 consecutive light/dark periods by indirect, open-circuit calorimetry using an Oxymax system (Columbus Instruments).

Tissue collection. After a 3-hour fast (9:00-12:00), mice were anesthetized with isoflurane (Abbott) and immediately sacrificed. Organs were weighed prior to fixation or cryopreservation in liquid nitrogen.

\section{Gene expression}

RNA was extracted using the RNeasy Mini Kit (Qiagen) or TRIzol reagent (Life Technologies) following homogenization with a Polytron (Kinematica) or FastPrep Lysing Matrix Tubes and Instrument (MP Biomedicals). Total RNA (1-5 $\mu \mathrm{g})$ was reverse transcribed using the MMLV Reverse Transcriptase kit (Invitrogen). cDNAs were measured by quantitative real-time PCR using Power SYBR Green mix and the StepOne Plus Sequence Detection System (Applied Biosystems) and were normalized using the housekeeping gene Rps29. Primers were designed using Primer Express software (Applied Biosystems) or were obtained from the University of Groningen database (http://www.labpediatricsrug.nl). The sequences of the primers used are provided in Supplemental Table 1.

\section{Histology}

Tissues were fixed in buffered formol fixative solution before dehydration and embedding in paraffin using a Leica ASP300S tissue processor (Leica). Five-micrometer sections prepared with a Microm HM 335 E microtome (Thermo Fisher Scientific) were stained with H\&E solution using classical procedures. Slides were then examined using a Nikon Eclipse $80 \mathrm{i}$ microscope with brightfield optics at $\times 20$ magnification before digital capture.

\section{Blood, tissues and stools dosages}

Blood samples were collected in heparin-coated tubes and immediately stored at $4^{\circ} \mathrm{C}$. Plasma was analyzed for total, HDL-, and LDL-cholesterol, and triglycerides using the Cobas C111 robot and appropriate reagents (Roche Diagnostics). Liver triglyceride content was determined using a colorimetric diagnostic kit (Diasys). Plasma insulin and leptin were measured using the mouse MADKMAG-71K metabolic kit (Merck-Millipore). Glucose levels were measured using an ACCU-CHEK Aviva monitor (Roche Diagnostics). For fecal caloric determination, stools were collected and dried at $60^{\circ} \mathrm{C}$ to a constant weight. Mean energy content was measured for each cage using a bomb calorimeter (IKA C200). The BAs were measured by isotope-dilution high performance liquid chromatography coupled to high resolution mass spectrometry (LC-MS). The assay was performed by combining the specimen $(50 \mu \mathrm{l})$ with the internal standards in methanol $(100 \mu \mathrm{l})$ and by adding $\mathrm{H}_{2} \mathrm{O}$ with $0.2 \%$ formic acid $(600 \mu \mathrm{l})$. Sample extraction and cleanup was performed using HLB SPE plates (Waters). LC-MS analysis was carried out using a Thermo Q-Exactive (Thermo Fisher Scientific). A volume of $10 \mu 1$ extract was injected into an Acquity UPLC HSS T* $1.8 \mu \mathrm{m}, 2.1 \times 100 \mathrm{~mm}$ column. Operating conditions of the MS were full-scan acquisition in negative mode ( $-\mathrm{H} \mathrm{m} / z$ of 370 to 522 , centroid acquisition, negative polarity). 


\section{Microbiota analysis}

Bacterial DNA was isolated and purified from feces recovered from the colon using the QIAmp DNA Stool Mini Kit (Qiagen). The phyla analysis following VCM administration was performed by quantitative real-time PCR, as previously reported (63). For in-depth analysis of the microbiota composition, the V3-V4 hypervariable region of the $16 \mathrm{~S}$ ribosomal gene was amplified by PCR (64). The amplified fragments were sequenced with the Miseq Reagent Kit v3 on an Illumina MiSeq machine producing paired-end 300-bp reads. The PANDAseq assembler was used to assemble raw paired reads into contigs (65). All contigs with any ambiguous nucleotides or with a length shorter than 390 nucleotides or longer than 450 nucleotides were removed from dataset. The reference operational taxonomic unit (OTU) sequences were identified using USEARCH software (version 8.1.1861) (66) after removing contig sequences that were not repeated at least 10 times across all samples. The abundance of OTUs in each sample library was computed using function search_global from USEARCH package (66). The 16S rRNA sequences with taxonomic annotation were obtained from the Silva database (67). All sequences with pintail quality score below 0.9 were removed from the 16S RNA sequence collection in order to avoid false taxonomic assignment related to the presence of chimeric sequences (68). The database was searched with the OTU reference sequences and the best hits with similarity exceeding $95 \%$ were used to assign taxonomy. The species-level assignment was kept only in case of sequences displaying nucleotide similarity above $99 \%$. All reference sequences that stayed unassigned were additionally classified using an online SINA alignment service with parameter minimal identity set to 0.9 (69). The Shannon diversity index was calculated using $\mathrm{R}$ package phyloseq (70). The ANOVA test followed by pairwise $t$ test was used to assess statistical significance of differences in the mean value of scores between groups. The $P$ values from pairwise comparison were adjusted using the Holm method. The clustering with UPGMA method and PCA of the data were performed using an unweighted UniFrac (71) distance matrix calculated with R package GUniFrac (72) based on the phylogenetic tree returned by the MUSCLE (73) program rooted with midpoint method with R package phangorn (74). The statistical significance of the difference in distribution of the Bacteroides/Firmicutes ratio in different groups was evaluated with the nonparametric Kruskal-Wallis rank-sum test and Wilcoxon rank-sum test as posthoc test for pairwise comparisons. The obtained $P$ values were adjusted using the Holm method. The differential abundance analysis of the phylum level was performed using a generalized linear model based on negative binomial distribution and the Wald test with R package DESeq2 (75). The probability values were corrected with the Benjamini-Hochberg procedure.

\section{Statistics}

Results are expressed as mean \pm SEM for the indicated number of observations. The unpaired 2-tailed Student's $t$ test and repeated-measures 1-way ANOVA were used when appropriate for comparison between groups of mice. $P$ less than 0.05 was considered statistically significant.

\section{Study approval}

Experimental protocols were performed in accordance with Swiss animal welfare laws (SCAV, Canton de Vaud).

\section{Author contributions}

ES and NP designed the research studies. ES, HH, SJB, SA, MA, and PDD conducted the experiments. ES, HH, SJB, MR, GPS, UA, MM, GG, and NP analyzed the data. KS, FRJ, and LF provided material and models. ES, AD, JSA, GPS, and NP wrote the manuscript. All authors were involved in editing the manuscript.

\section{Acknowledgments}

We thank Nadia Preitner and Jean-Pierre Rey for their excellent technical assistance, Frédéric Preitner, Gilles Willemin, and Anabela Cristina Da Costa (UNIL Mouse Metabolic Evaluation Facility) for scientific and technical expertise in biological dosages; the staff of the Bugnon 7 animal facility (Alain Gnecchi, Daniel Catalano, Duarte De Azevedo, Fanny Thévenaz, and Aurélie Chantany) as well as Laure Seriot and Gisèle Ferrand for their valuable help concerning animal maintenance; and Jean-Christophe Stehle and the staff of the CHUV Mouse Pathology Facility for their deep expertise in histology. We thank Luc Pellerin for sharing qPCR machines. We also thank Justine Bouilly, Daniele Cassatella, Sara Santini, Andrea 
Messina, Nicolas Niederländer, Cheng Xu, Panos Ziros, and Isabel Lopez Mejia for scientific interactions and support during the project. The N.P. and K.S. labs are part of the Lausanne Integrative Metabolism \& Nutrition Alliance (LIMNA). This work was supported by grants from the Swiss National Foundation (Synergia grant CRSII3-141960 to N.P., U.A., and M.M.; SNF grant 31003A-153328 to N.P.) and by grants from the National Institute of Health (NIDCR grant DE13686 to M.M.). This work was also supported by the Novartis Foundation for Medical-Biological Research (grant 16A027 to N.P.).

Address correspondence to: Emmanuel Somm, Service of Endocrinology, Diabetology and Metabolism (Lausanne University Hospital)/Faculty of Biology and Medicine (University of Lausanne), Département de Physiologie, rue du Bugnon 7, 1005 Lausanne, Switzerland. Phone: 00.41.21.692.5517; E-mail: emmanuel.somm@chuv.ch. Or to: Nelly Pitteloud, Service of Endocrinology, Diabetology and Metabolism (Lausanne University Hospital)/Faculty of Biology and Medicine (University of Lausanne), Hôtel des Patients (Niveau 2), Avenue de la Sallaz 8, 1011 Lausanne, Switzerland. Phone: 00.41.21.314.8796; E-mail: nelly.pitteloud@chuv.ch.

1. Ito S, et al. Molecular cloning and expression analyses of mouse betaklotho, which encodes a novel Klotho family protein. Mech Dev. 2000;98(1-2):115-119.

2. Kharitonenkov A, et al. FGF-21/FGF-21 receptor interaction and activation is determined by betaKlotho. J Cell Physiol. 2008;215(1):1-7.

3. Ogawa Y, et al. BetaKlotho is required for metabolic activity of fibroblast growth factor 21. Proc Natl Acad Sci USA. 2007;104(18):7432-7437.

4. Owen BM, Mangelsdorf DJ, Kliewer SA. Tissue-specific actions of the metabolic hormones FGF15/19 and FGF21. Trends Endocrinol Metab. 2015;26(1):22-29.

5. Kharitonenkov A, et al. FGF-21 as a novel metabolic regulator. J Clin Invest. 2005;115(6):1627-1635.

6. Inagaki T, et al. Endocrine regulation of the fasting response by PPARalpha-mediated induction of fibroblast growth factor 21. Cell Metab. 2007;5(6):415-425.

7. Fisher FM, et al. FGF21 regulates PGC-1 $\alpha$ and browning of white adipose tissues in adaptive thermogenesis. Genes Dev. 2012;26(3):271-281.

8. Hondares E, et al. Thermogenic activation induces FGF21 expression and release in brown adipose tissue. J Biol Chem. 2011;286(15):12983-12990.

9. Adams AC, Cheng CC, Coskun T, Kharitonenkov A. FGF21 requires ßklotho to act in vivo. PLoS ONE. 2012;7(11):e49977.

10. Ding X, et al. $\beta$ Klotho is required for fibroblast growth factor 21 effects on growth and metabolism. Cell Metab. 2012;16(3):387-393.

11. Bookout AL, et al. FGF21 regulates metabolism and circadian behavior by acting on the nervous system. Nat Med. 2013;19(9):1147-1152.

12. Owen BM, et al. FGF21 contributes to neuroendocrine control of female reproduction. Nat Med. 2013;19(9):1153-1156.

13. Owen BM, et al. FGF21 acts centrally to induce sympathetic nerve activity, energy expenditure, and weight loss. Cell Metab. 2014;20(4):670-677.

14. Kurosu H, et al. Tissue-specific expression of betaKlotho and fibroblast growth factor (FGF) receptor isoforms determines metabolic activity of FGF19 and FGF21. J Biol Chem. 2007;282(37):26687-26695.

15. Lin BC, Wang M, Blackmore C, Desnoyers LR. Liver-specific activities of FGF19 require Klotho beta. J Biol Chem. 2007;282(37):27277-27284.

16. Inagaki T, et al. Fibroblast growth factor 15 functions as an enterohepatic signal to regulate bile acid homeostasis. Cell Metab. 2005;2(4):217-225.

17. Ito S, Fujimori T, Furuya A, Satoh J, Nabeshima Y, Nabeshima Y. Impaired negative feedback suppression of bile acid synthesis in mice lacking betaKlotho. J Clin Invest. 2005;115(8):2202-2208.

18. Kobayashi K, et al. Hepatocyte $\beta$-Klotho regulates lipid homeostasis but not body weight in mice. FASEB J. 2016;30(2):849-862

19. Watanabe M, et al. Bile acids induce energy expenditure by promoting intracellular thyroid hormone activation. Nature. 2006;439(7075):484-489.

20. Begley M, Gahan CG, Hill C. The interaction between bacteria and bile. FEMS Microbiol Rev. 2005;29(4):625-651.

21. Miyata M, Takamatsu Y, Kuribayashi H, Yamazoe Y. Administration of ampicillin elevates hepatic primary bile acid synthesis through suppression of ileal fibroblast growth factor 15 expression. J Pharmacol Exp Ther. 2009;331(3):1079-1085.

22. Sayin SI, et al. Gut microbiota regulates bile acid metabolism by reducing the levels of tauro-beta-muricholic acid, a naturally occurring FXR antagonist. Cell Metab. 2013;17(2):225-235.

23. Hildebrandt MA, et al. High-fat diet determines the composition of the murine gut microbiome independently of obesity. Gastroenterology. 2009;137(5):1716-24.e1.

24. Ley RE, Bäckhed F, Turnbaugh P, Lozupone CA, Knight RD, Gordon JI. Obesity alters gut microbial ecology. Proc Natl Acad Sci USA. 2005;102(31):11070-11075.

25. Turnbaugh PJ, Ley RE, Mahowald MA, Magrini V, Mardis ER, Gordon JI. An obesity-associated gut microbiome with increased capacity for energy harvest. Nature. 2006;444(7122):1027-1031.

26. Ridlon JM, Kang DJ, Hylemon PB. Bile salt biotransformations by human intestinal bacteria. J Lipid Res. 2006;47(2):241-259.

27. Badman MK, Koester A, Flier JS, Kharitonenkov A, Maratos-Flier E. Fibroblast growth factor 21-deficient mice demonstrate impaired adaptation to ketosis. Endocrinology. 2009;150(11):4931-4940. 
28. Hotta Y, et al. Fibroblast growth factor 21 regulates lipolysis in white adipose tissue but is not required for ketogenesis and triglyceride clearance in liver. Endocrinology. 2009;150(10):4625-4633.

29. Liang Q, et al. FGF21 maintains glucose homeostasis by mediating the cross talk between liver and brain during prolonged fasting. Diabetes. 2014;63(12):4064-4075.

30. Potthoff MJ, et al. FGF21 induces PGC-1alpha and regulates carbohydrate and fatty acid metabolism during the adaptive starvation response. Proc Natl Acad Sci USA. 2009;106(26):10853-10858.

31. Markan KR, et al. Circulating FGF21 is liver derived and enhances glucose uptake during refeeding and overfeeding. Diabetes. 2014;63(12):4057-4063.

32. Ge H, et al. Fibroblast growth factor receptor 4 (FGFR4) deficiency improves insulin resistance and glucose metabolism under diet-induced obesity conditions. J Biol Chem. 2014;289(44):30470-30480.

33. de Aguiar Vallim TQ, et al. MAFG is a transcriptional repressor of bile acid synthesis and metabolism. Cell Metab. 2015;21(2):298-310.

34. Watanabe $\mathrm{M}$, et al. Bile acid binding resin improves metabolic control through the induction of energy expenditure. PLoS ONE. 2012;7(8):e38286

35. Maruyama T, et al. Identification of membrane-type receptor for bile acids (M-BAR). Biochem Biophys Res Commun. 2002;298(5):714-719.

36. de Aguiar Vallim TQ, Tarling EJ, Edwards PA. Pleiotropic roles of bile acids in metabolism. Cell Metab. 2013;17(5):657-669.

37. Kuipers F, Bloks VW, Groen AK. Beyond intestinal soap--bile acids in metabolic control. Nat Rev Endocrinol. 2014;10(8):488-498.

38. Wahlström A, Sayin SI, Marschall HU, Bäckhed F. Intestinal crosstalk between bile acids and microbiota and its impact on host metabolism. Cell Metab. 2016;24(1):41-50.

39. Joyce SA, et al. Regulation of host weight gain and lipid metabolism by bacterial bile acid modification in the gut. Proc Natl Acad Sci USA. 2014;111(20):7421-7426.

40. Li F, et al. Microbiome remodelling leads to inhibition of intestinal farnesoid X receptor signalling and decreased obesity. Nat Commun. 2013;4:2384

41. Parséus A, et al. Microbiota-induced obesity requires farnesoid X receptor. Gut. 2017;66(3):429-437.

42. Vrieze A, et al. Impact of oral vancomycin on gut microbiota, bile acid metabolism, and insulin sensitivity. $J$ Hepatol. 2014;60(4):824-831

43. Brown AJ, et al. The orphan G protein-coupled receptors GPR41 and GPR43 are activated by propionate and other short chain carboxylic acids. J Biol Chem. 2003;278(13):11312-11319.

44. Kimura I, et al. The gut microbiota suppresses insulin-mediated fat accumulation via the short-chain fatty acid receptor GPR43 Nat Commun. 2013;4:1829.

45. Jiang C, et al. Intestinal farnesoid X receptor signaling promotes nonalcoholic fatty liver disease. J Clin Invest. 2015;125(1):386-402.

46. Jiang C, et al. Intestine-selective farnesoid X receptor inhibition improves obesity-related metabolic dysfunction. Nat Commun 2015;6:10166.

47. Arab JP, Karpen SJ, Dawson PA, Arrese M, Trauner M. Bile acids and nonalcoholic fatty liver disease: Molecular insights and therapeutic perspectives. Hepatology. 2017;65(1):350-362.

48. Itoh N, Nakayama Y, Konishi M. Roles of FGFs as paracrine or endocrine signals in liver development, health, and disease Front Cell Dev Biol. 2016;4:30.

49. Badman MK, Pissios P, Kennedy AR, Koukos G, Flier JS, Maratos-Flier E. Hepatic fibroblast growth factor 21 is regulated by PPARalpha and is a key mediator of hepatic lipid metabolism in ketotic states. Cell Metab. 2007;5(6):426-437.

50. Kim SH, et al. Fibroblast growth factor 21 participates in adaptation to endoplasmic reticulum stress and attenuates obesityinduced hepatic metabolic stress. Diabetologia. 2015;58(4):809-818.

51. Huang X, Yang C, Luo Y, Jin C, Wang F, McKeehan WL. FGFR4 prevents hyperlipidemia and insulin resistance but underlies high-fat diet induced fatty liver. Diabetes. 2007;56(10):2501-2510.

52. Li T, et al. Overexpression of cholesterol $7 \alpha$-hydroxylase promotes hepatic bile acid synthesis and secretion and maintains cholesterol homeostasis. Hepatology. 2011;53(3):996-1006.

53. Watanabe M, et al. Bile acids lower triglyceride levels via a pathway involving FXR, SHP, and SREBP-1c. J Clin Invest. 2004;113(10):1408-1418.

54. Díaz-Delfín J, Hondares E, Iglesias R, Giralt M, Caelles C, Villarroya F. TNF- $\alpha$ represses $\beta$-Klotho expression and impairs FGF21 action in adipose cells: involvement of JNK1 in the FGF21 pathway. Endocrinology. 2012;153(9):4238-4245.

55. Zhao Y, et al. IL-1 $\beta$ inhibits $\beta$-Klotho expression and FGF19 signaling in hepatocytes. Am J Physiol Endocrinol Metab. 2016;310(4):E289-E300.

56. Singhal G, et al. Fibroblast growth factor 21 (FGF21) protects against high fat diet induced inflammation and islet hyperplasia in pancreas. PLoS One. 2016;11(2):e0148252.

57. Lee MS, et al. Fibroblast growth factor-21 protects human skeletal muscle myotubes from palmitate-induced insulin resistance by inhibiting stress kinase and NF-кB. Metab Clin Exp. 2012;61(8):1142-1151.

58. Liu MH. FGF-21 alleviates diabetes-associated vascular complications: Inhibiting NF-кB/NLRP3 inflammasome-mediated inflammation? Int J Cardiol. 2015;185:320-321.

59. Fisher FM, et al. Fibroblast growth factor 21 limits lipotoxicity by promoting hepatic fatty acid activation in mice on methionine and choline-deficient diets. Gastroenterology. 2014;147(5):1073-83.e6.

60. Tanaka N, et al. Role of fibroblast growth factor 21 in the early stage of NASH induced by methionine- and choline-deficient diet. Biochim Biophys Acta. 2015;1852(7):1242-1252.

61. Broeders EP, et al. The bile acid chenodeoxycholic acid increases human brown adipose tissue activity. Cell Metab. $2015 ; 22(3): 418-426$

62. Thomas C, et al. TGR5-mediated bile acid sensing controls glucose homeostasis. Cell Metab. 2009;10(3):167-177.

63. Bacchetti De Gregoris T, Aldred N, Clare AS, Burgess JG. Improvement of phylum- and class-specific primers for real-time 
PCR quantification of bacterial taxa. J Microbiol Methods. 2011;86(3):351-356.

64. Klindworth A, et al. Evaluation of general 16S ribosomal RNA gene PCR primers for classical and next-generation sequencingbased diversity studies. Nucleic Acids Res. 2013;41(1):e1.

65. Masella AP, Bartram AK, Truszkowski JM, Brown DG, Neufeld JD. PANDAseq: paired-end assembler for illumina sequences. BMC Bioinformatics. 2012;13:31.

66. Edgar RC. UPARSE: highly accurate OTU sequences from microbial amplicon reads. Nat Methods. 2013;10(10):996-998.

67. Quast C, et al. The SILVA ribosomal RNA gene database project: improved data processing and web-based tools. Nucleic Acids Res. 2013;41(Database issue):D590-D596.

68. Ashelford KE, Chuzhanova NA, Fry JC, Jones AJ, Weightman AJ. At least 1 in 20 16S rRNA sequence records currently held in public repositories is estimated to contain substantial anomalies. Appl Environ Microbiol. 2005;71(12):7724-7736.

69. Pruesse E, Peplies J, Glöckner FO. SINA: accurate high-throughput multiple sequence alignment of ribosomal RNA genes. Bioinformatics. 2012;28(14):1823-1829.

70. McMurdie PJ, Holmes S. phyloseq: an R package for reproducible interactive analysis and graphics of microbiome census data. PLoS One. 2013;8(4):e61217.

71. Lozupone C, Knight R. UniFrac: a new phylogenetic method for comparing microbial communities. Appl Environ Microbiol. 2005;71(12):8228-8235.

72. Chen J, et al. Associating microbiome composition with environmental covariates using generalized UniFrac distances. Bioinformatics. 2012;28(16):2106-2113.

73. Edgar RC. MUSCLE: multiple sequence alignment with high accuracy and high throughput. Nucleic Acids Res. 2004;32(5):17921797.

74. Schliep KP. phangorn: phylogenetic analysis in R. Bioinformatics. 2011;27(4):592-593.

75. Love MI, Huber W, Anders S. Moderated estimation of fold change and dispersion for RNA-seq data with DESeq2. Genome Biol. 2014;15(12):550. 